\title{
Phoneutria nigriventer venom: A pharmacological treasure
}

\author{
Steve Peigneur ${ }^{\mathrm{a}, \mathrm{b}}$, Maria Elena de Lima ${ }^{\mathrm{b}, \mathrm{c}, *}$, Jan Tytgat ${ }^{\mathrm{a}, * *}$ \\ a Toxicology and Pharmacology, University of Leuven (KU Leuven), Campus Gasthuisberg, PO Box 922, Herestraat 49, 3000 Leuven, Belgium \\ ${ }^{\mathrm{b}}$ Laboratório de Venenos e Toxinas Animais, Dept de Bioquímica e Imunologia, Instituto de Ciências Biológicas, Universidade Federal de Minas Gerais, 31270-901, Belo- \\ Horizonte, $M G$, Brazil \\ ${ }^{\mathrm{c}}$ Programa de Pós-graduação em Ciências da Saúde, Biomedicina e Medicina, Instituto de Ensino e Pesquisa da Santa Casa de Belo Horizonte, Grupo Santa Casa de Belo \\ Horizonte, Belo Horizonte, MG, Brazil
}

\section{A B S T R A C T}

In millions of years, spiders have optimized their venoms in order to assure successful prey capture and defence against predators. Spider venoms have become unique cocktails of biological active components enabling potentially interesting application for drug discovery or for agricultural purposes. The venom of Phoneutria nigriventer has been studied for over 60 years. This spider is responsible for a high number of envenomations with severe clinical manifestations in humans, which necessitates a comprehensive knowledge of its venom composition. With over 40 different neurotoxic peptides characterized so far and still many more awaiting identification, this venom is undoubtedly a pharmacological treasure. This review provides an overview of the Phoneutria nigriventer toxins known today and describes their mechanism of action at a molecular level. We critically discuss the potential of the Phoneutria nigriventer venom peptides as pharmaceutical tools or lead compounds for drug development.

\section{Spider venom as a source of drug discovery}

Spiders can be considered as one of the most successful venomous animals ever to habitat the planet. So far over 47000 species have been characterized (Pineda et al., 2014). According to ArachnoServer, only of approximately 100 species the venom has been studied. This has resulted in 1427 characterized spider venom peptides (Herzig et al., 2011; Pineda et al., 2017). Since it is believed that spider venoms contain over 10 million biologically active peptides, no more than $0.01 \%$ of spider peptides have thus been studied so far (Escoubas, 2006; Klint et al., 2012). Therefore, spider venom can be considered as untapped treasure of biological active compounds that are potentially interesting for drug discovery as well as for the development of bioinsecticides to control pests. Up to date, no spider peptide-based drug has been approved by the Food and Drug Administration. However, one should take into account the small number of spider venom peptides investigated so far. This is a significant lower number compared for instance with scorpion or snake venom components. Several spider venom peptides have been characterized as insecticidal peptides with a strong preference for insect over mammalian targets. Illustrative hereof is the spider toxin $\omega / \kappa-H X T V-H v 1 a$. This peptide, isolated from the venom of Hadronyche versuta, is a potent inhibitor of insect voltagegated calcium (Cav) channels devoid of activity on mammalian Cav channels. A modified synthetic version of this toxin is the basis of the approved insecticidal agent SPEAR ${ }^{\circ}$ (Fitches et al., 2012; Herzig and King, 2015; King and Hardy, 2013). Recently, exciting research has also highlighted the potential of spider venom-based drug discovery. Hi1a, a toxin isolated from the Australian funnel-web spider Hadronyche infensa, was found to be highly neuroprotective in acid-mediated neuronal injuries. This toxin inhibits acid-sensing ion channel 1a and hereby strongly attenuates for instance brain damage after stroke (Chassagnon et al., 2017). Hila is thus considered as a lead for development of therapeutics to protect the brain from ischemic injury.

\section{Phoneutria nigriventer}

The spiders of the genus Phoneutria are members of the family Ctenidae, suborder Labidognatha, and order Araneidae. They inhabit forests of the neotropical region from Southern Central America (Costa Rica) throughout South America, from the East of the Andes to the North of Argentina. The Phoneutria spiders are also known as the "armed spider" because of the characteristic posture they adopt when threatened. When in defence position, the Phoneutria will stand on his hind legs while typically raising four frontal legs up high, resulting in an "armed position" (Fig. 1). Another popular name is the "banana spider" which relates to their preference for hiding in banana bunches.

\footnotetext{
* Corresponding author. Programa de Pós-graduação em Ciências da Saúde, Biomedicina e Medicina, Instituto de Ensino e Pesquisa da Santa Casa de Belo Horizonte, Grupo Santa Casa de Belo Horizonte, Belo Horizonte, MG, Brazil.

** Corresponding author. Toxicology and Pharmacology, University of Leuven (KU Leuven), Campus Gasthuisberg, PO Box 922 , Herestraat 49, 3000 Leuven, Belgium.

E-mail addresses: lima.mariaelena@gmail.com (M.E. de Lima), jan.tytgat@kuleuven.be (J. Tytgat).
} 


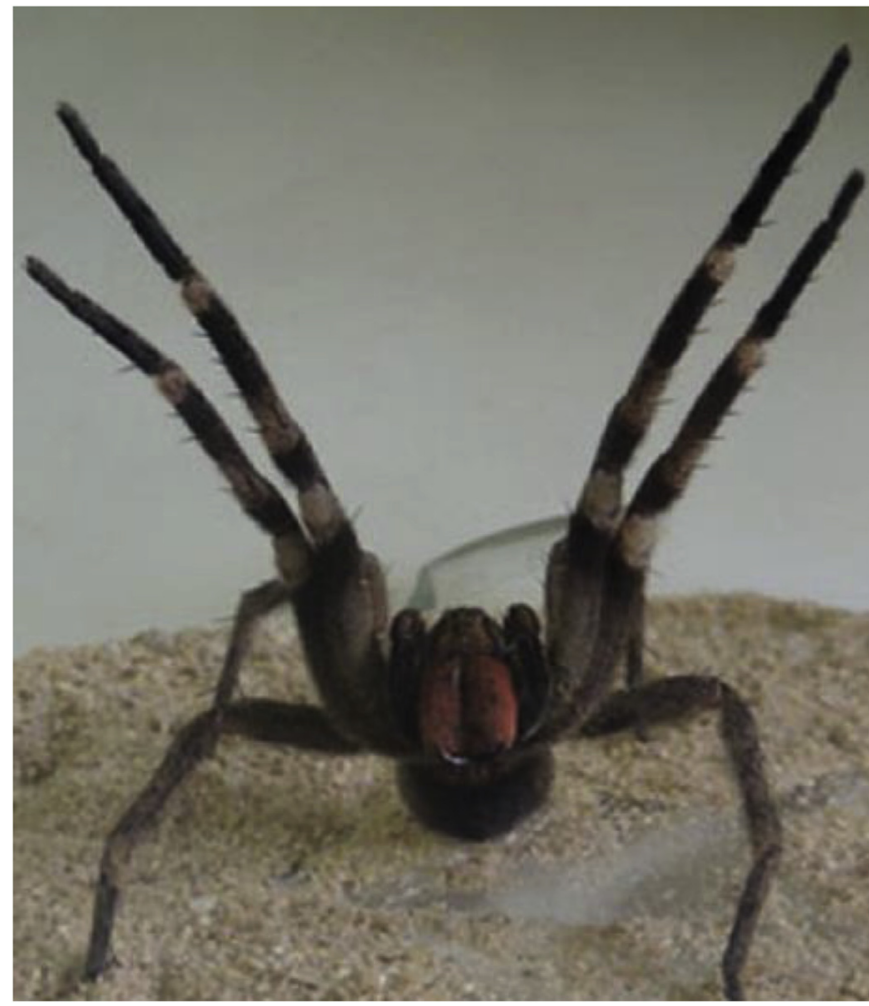

Fig. 1. Phoneutria nigriventer, the "armed spider". The picture shows the spider assuming the typical defensive or "armed" position. (Photographed by Alcides Sousa, at Fundacão Ezequiel Dias, Belo Horizonte, Minas Gerais, Brazil).

The genus Phoneutria belongs to the Retrolateral Tibial Apophysis (RTA) clade, whose adaptive and evolutionary process is associated with the loss of cribellate silk and prey-capture webs. They only use silk for the production of the sacs in which the eggs hatch or for nursery webs. These are wandering spiders with nocturnal habits. They are active hunters, relying on their fast acting and efficient venom for prey capture and defence. Their natural preys are insects although there are reports of Phoneutria hunting on other spiders and small rodents as well (de Lima et al., 2016; Herzig et al., 2002).

Phoneutria nigriventer are very aggressive, solitary spiders. They are synanthropic species, explaining the high number of human accidents occurring with this spider. Human evenomations involving Phoneutria spiders occur mainly in Brazil, but there are reports of sporadic cases in Central America and in neighbouring countries (de Lima et al., 2016). More recently, the export of bananas to Europe has resulted in certain cases of Phoneutria envenomation in countries such as the United Kingdom and the Netherlands. Most accidents involving humans are mild with only up to $0.5 \%$ of severe cases (Hauke and Herzig, 2017). Despite the venom being highly neurotoxic, the amount inoculated through the bite is usually too small to induce lethal effects. However, fatal envenomation usually occurs after a bite by female species. It is reported that females inject a larger amount of venom compared to males. Furthermore, as with most venomous animals, intersexual differences in venom composition have been demonstrated (Herzig et al., 2002). The clinical manifestations of severe systemic intoxication are usually seen in elderly and children. In such cases, the penile erection or priapism is one of the most noted signs of phoneutrism. Other clinical manifestations often reported after a Phoneutria bite are convulsions, agitation, somnolence, nausea, profuse sweating and vomiting, lacrimation, excessive salivation, hypertension, tachycardia, tachypnea, tremors and spastic paralysis (de Lima et al., 2016; Raposo et al., 2016). Cases of systemic poisoning in adults are uncommon but may happen. The effects observed in experimental animals after venom injection are very similar to those observed in humans after the accidents with this spider. An interesting case report is the envenomation of a 52-year-old man who suffered from a neck bite by a female specimen of Phoneutria nigriventer. The patient experienced intense local pain, episodes of vomiting, tremors, blurred vision and excessive sweating. After $2 \mathrm{~h}$, the patient was treated with captopril and meperidine because of a strongly elevated blood pressure. This treatment allowed stabilization of blood pressure and heart rate. Nevertheless, tachypnea, gentle shaking, cold extremities, profuse sweating, generalized tremors, and priapism subsisted. Treatment with antivenom resulted in complete recovery within $1 \mathrm{~h}$ (Bucaretchi et al., 2008). This case report nicely demonstrated the presence of venom components with a strong neurotoxic activity, and herewith, underlined the potential pharmaceutical applicability of Phoneutria venom. It is thus of no surprise that the venom of $P$. nigriventer is considered as a pharmacological treasure for drug discovery for over 60 years now. Indeed, this venom is a complex mixture of proteins and peptides, including neurotoxins, acting on ion channels and chemical receptors of the nervous and muscular systems of insects and mammals (Richardson et al., 2006). Notwithstanding that no Phoneutria venom-derived peptide has made it to the drug market, the potential pharmacological applicability of these peptides is evidenced by the ample research studies using these peptides, not only as potent ligands for specific targets, but also as tools to have a better understanding of their physiological function and their involvement in diseases and channelopathies.

The first studies on $P$. nigriventer venom, report the presence of biologically active proteins such as peptides, proteases, and hyaluronidase. Furthermore, other active compounds such as histamine serotonin and some free amino acids were also identified (de Lima et al., 2016; Gomez et al., 2002). Early work indicated that the whole venom exhibits a pronounced neurotoxic activity. Injection of whole or partially fractionized venom caused a myriad of excitatory symptoms in experimental animals (Bucherl, 1953, 1969; Schenberg and Lima, 1966). These observations in animals corroborated well with the reports on human envenomation (Bucaretchi et al., 2008; Diniz et al., 1990). The very first biochemical and pharmacological characterization of an isolated Phoneutria nigriventer toxin was performed in 1990s by Dr. Diniz and colleagues (Rezende et al., 1991) at Fundação Ezequiel Dias (Belo Horizonte, MG, Brazil). Almost 30 years before, Diniz has been the pioneer in studying this venom (Diniz, 1963). Nowadays P. nigriventer venom has been extensively studied making it one of the most studied spider venoms in the world. Besides some non-protein lowmolecular-mass compounds, 41 neurotoxins have been identified from the crude venom up to date (Tables 1 and 2) (Herzig et al., 2011; Pineda et al., 2017). The $P$. nigriventer venom (PNV) has also been studied for its potential ability to cross the blood-brain barrier. This has recently been very nicely reviewed (Cruz-Hofling et al., 2016). This review shows that certain components of the PNV cause activation in multiple brain areas and upregulate the expression of vascular endothelial growth factor (VEGF) and its receptors Fms-like tyrosine kinase1 (Flt-1) and fetal liver kinase 1 (Flk-1). The authors suggested that these data of the PNV mechanism in the CNS can contribute to improving the treatment in cases of phoneutrism and, in addition, that PNV could be a potential tool for studies on drug permeability across the BBB.

\section{Nomenclature of Phoneutria nigriventer toxins}

The nomenclature of Phoneutria nigriventer toxins is rather confusing and problematic. Over time, often several names have been given to the same peptide. Historically, the Phoneutria toxins are annotated based on their occurrence in the venom when following the venom purification methods used in the first studies (Diniz et al., 1990), i.e. based on a particular chromatographic step and in the order of elution of the toxin, in this step. In an attempt to solve the confusing nomenclature of peptides of spiders and of other animal venoms, King et al. (2008) have proposed a rational nomenclature, which considers the molecular target 


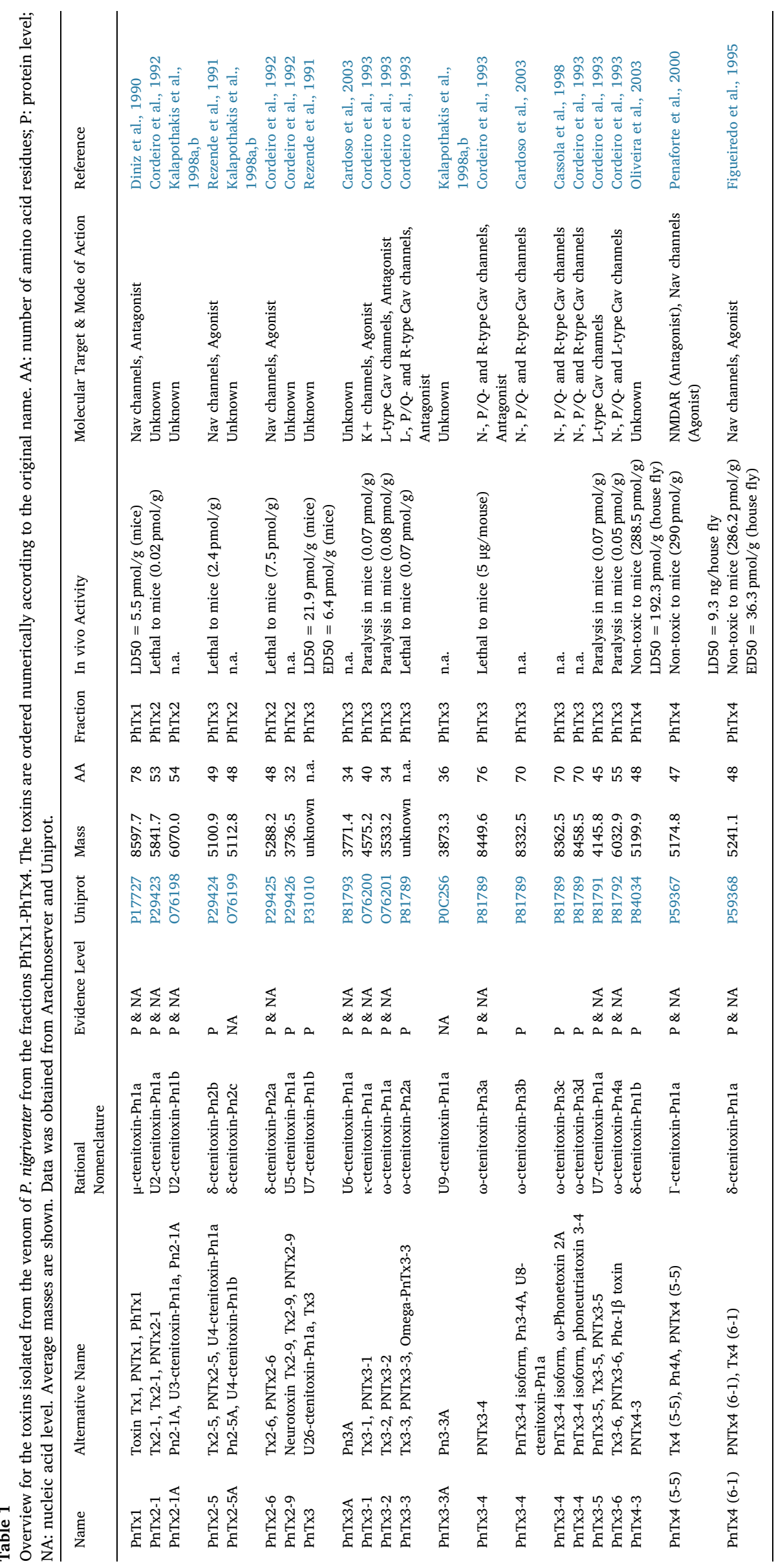


Table 2

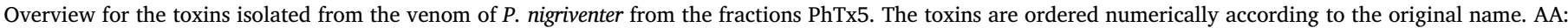

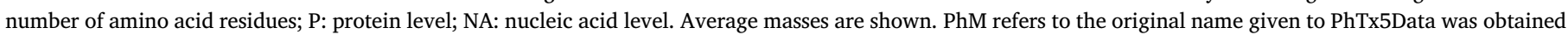
from Arachnoserver and Uniprot.

\begin{tabular}{|c|c|c|c|c|c|c|c|c|}
\hline Name & Alternative Name & Rational Nomenclature & Evidence Level & Uniprot & Mass & AA & In vivo Activity & Reference \\
\hline Pn3-5A & & U10-ctenitoxin-Pn1a & NA & P0C2S9 & 4705.3 & 39 & n.a. & Cardoso et al., 2003 \\
\hline Pn3-6A & & U11-ctenitoxin-Pn1a & NA & P0C2S7 & 6589.4 & 58 & n.a. & Cardoso et al., 2003 \\
\hline PNTx22C3 & & U11-ctenitoxin-Pn1b & $\mathrm{P}$ & P84011 & 6571.3 & 58 & Non-toxic to mice & Richardson et al., 2006 \\
\hline Pn3-6B & & U12-ctenitoxin-Pn1a & NA & P0C2S8 & 6370.3 & 58 & n.a. & Cardoso et al., 2003 \\
\hline PNTx13C3 & & U13-ctenitoxin-Pn1a & $\mathrm{P}$ & P83894 & 3552.1 & 32 & n.a. & Richardson et al., 2006 \\
\hline PNTx24A0C3 & & U13-ctenitoxin-Pn1b & $\mathrm{P}$ & P84017 & 3481.0 & 31 & n.a. & Richardson et al., 2006 \\
\hline PNTx24A0C4 & & U13-ctenitoxin-Pn1c & $\mathrm{P}$ & P84018 & 3679.3 & 33 & n.a. & Richardson et al., 2006 \\
\hline PNTx22A0C1 & & U14-ctenitoxin-Pn1a & $\mathrm{P}$ & P83998 & 4079.9 & 35 & Non-toxic to mice and housefly & Richardson et al., 2006 \\
\hline PNTx27C4 & & U17-ctenitoxin-Pn1a & $\mathrm{P}$ & P83996 & 4062.8 & 36 & Lethal in mice ( $3.0 \mu \mathrm{g} /$ mouse) & Richardson et al., 2006 \\
\hline PNTx30C3 & & U18-ctenitoxin-Pn1a & $\mathrm{P}$ & P83999 & 7876.6 & n.a. & Lethal in mice ( $3.0 \mu \mathrm{g} /$ mouse $)$ & Richardson et al., 2006 \\
\hline PNTx16C1 & & U19-ctenitoxin-Pn1a & $\mathrm{P}$ & P83997 & 7577.4 & 68 & n.a. & Richardson et al., 2006 \\
\hline Pn4B & & U1-ctenitoxin-Pn1a & NA & P61229 & 5815.4 & 47 & n.a. & Penaforte et al., 2000 \\
\hline PNTx22C5 & & U20-ctenitoxin-Pn1a & $\mathrm{P}$ & P84093 & 8855.0 & 80 & Lethal in mice $(3.0 \mu \mathrm{g} /$ mouse $)$ & Richardson et al., 2006 \\
\hline PN47 & & U21-ctenitoxin-Pn1a & $\mathrm{P}$ & P84033 & 22089.0 & 245 & n.a. & Richardson et al., 2006 \\
\hline PN10C5 & & U23-ctenitoxin-Pn1a & $P$ & P84015 & 3672.7 & 33 & n.a. & Richardson et al., 2006 \\
\hline PN16C3 & & U24-ctenitoxin-Pn1a & $\mathrm{P}$ & P84032 & 14775.7 & 128 & n.a. & Richardson et al., 2006 \\
\hline PnV2 & & U27-ctenitoxin-Pn1a & $\mathrm{P}$ & Q9TWR5 & unknown & n.a. & n.a. & Marangoni et al., 1993 \\
\hline PnV1 & & U28-ctenitoxin-Pn1a & $\mathrm{P}$ & Q7M3P1 & unknown & n.a. & n.a. & Richardson et al., 2006 \\
\hline PnTkP-I & tachykinin peptide-I & U29-ctenitoxin-Pn1a & $\mathrm{P}$ & P86298 & 872.0 & 7 & n.a. & Pimenta et al., 2005 \\
\hline PnTkP-II & tachykinin peptide-II & U29-ctenitoxin-Pn1b & $\mathrm{P}$ & P86299 & 1000.0 & 8 & n.a. & Pimenta et al., 2005 \\
\hline PnTkP-III & tachykinin peptide-III & U29-ctenitoxin-Pn1c & $\mathrm{P}$ & P86300 & 1028.0 & 8 & n.a. & Pimenta et al., 2005 \\
\hline PnTkP-IV & tachykinin peptide-IV & U29-ctenitoxin-Pn1d & $\mathrm{P}$ & P86301 & 1147.3 & 9 & n.a. & Pimenta et al., 2005 \\
\hline PnTkP-V & tachykinin peptide-V & U29-ctenitoxin-Pn1e & $\mathrm{P}$ & P86302 & 1175.4 & 8 & n.a. & Pimenta et al., 2005 \\
\hline PnTkP-VI & tachykinin peptide-VI & U29-ctenitoxin-Pn1f & $\mathrm{P}$ & P86303 & 1217.5 & 10 & n.a. & Pimenta et al., 2005 \\
\hline PnTkP-VII & tachykinin peptide-VII & U29-ctenitoxin-Pn1g & $\mathrm{P}$ & P86304 & 1232.8 & 10 & n.a. & Pimenta et al., 2005 \\
\hline PnTkP-VIII & tachykinin peptide-VIII & U29-ctenitoxin-Pn1h & $\mathrm{P}$ & P86305 & 1337.9 & 10 & n.a. & Pimenta et al., 2005 \\
\hline PnTkP-IX & tachykinin peptide-IX & U29-ctenitoxin-Pn1i & $\mathrm{P}$ & P86306 & 1509.9 & 12 & n.a. & Pimenta et al., 2005 \\
\hline PnTkP-X & tachykinin peptide-X & U29-ctenitoxin-Pn1j & $\mathrm{P}$ & P86307 & 1610.1 & 13 & n.a. & Pimenta et al., 2005 \\
\hline PnTkP-XI & tachykinin peptide-XI & U29-ctenitoxin-Pn1k & $\mathrm{P}$ & P86308 & 1623.9 & 13 & n.a. & Pimenta et al., 2005 \\
\hline PnTkP-XII & tachykinin peptide-XII & U29-ctenitoxin-Pn11 & $\mathrm{P}$ & P86309 & 1626.4 & 13 & n.a. & Pimenta et al., 2005 \\
\hline PnTkP-XIII & tachykinin peptide-XIII & U29-ctenitoxin-Pn1m & $\mathrm{P}$ & P86310 & 1637.9 & 13 & n.a. & Pimenta et al., 2005 \\
\hline PnTkP-XIV & tachykinin peptide-XIV & U29-ctenitoxin-Pn1n & $\mathrm{P}$ & P86311 & 1653.8 & 13 & n.a. & Pimenta et al., 2005 \\
\hline PnTkP-XV & tachykinin peptide-XVI & U29-ctenitoxin-Pn1o & $\mathrm{P}$ & P86297 & 1653.9 & 14 & n.a. & Pimenta et al., 2005 \\
\hline PhM1 & tachykinin peptide PhM1 & U29-ctenitoxin-Pn1p & $\mathrm{P}$ & n.a. & 1346.6 & 11 & n.a. & Pimenta et al., 2005 \\
\hline
\end{tabular}

(including sub-types) of the toxin and also the family, genus and species of the animal from which the toxin was obtained. As an example, the toxin originally named Tx2-6/PnTx2-6 from P.nigriventer, is also annotated, following the new nomenclature as: $\delta$-CNTX-Pn2a (delta meaning its modulating activity on Nav inactivation, CNTX = Ctenitoxin from Ctenidae family; $\mathrm{Pn}=$ Phoneutria nigriventer, $=$ isoform). Some efforts have been done to adopt the new nomenclature of King. However, in general with Phoneutria venom peptides, the name given to the toxin in the first publication has become the common used name of the toxin. Therefore, in this review we adopted the name as found in the respective publications. The corresponding names suggested by King's nomenclature can be easily consulted in ArachoServer and in Tables 1 and 2 Since the venom is a complex mixture, an efficient purification method was needed. Therefore, an activity-guided purification and fractionation procedure, using gel filtration and reversed-phase chromatography had to be developed (Diniz et al., 1990). Following this procedure typically generated five distinct venom fractions. Each fraction showed different activity when assayed in mammals and/or insects, suggesting the presence of toxins with different pharmacological properties (Figueiredo et al., 1995; Rezende et al., 1991). For most of the initial studies, toxicity was evaluated in vivo by intracerebral (i.c.) or intrathoracic injections in mice and insects, respectively, and in vitro by smooth muscle assays using guinea pig ileum, besides some assays in synaptosomes from rat brain. Using this activity guided purification method, four distinct venom fractions, named PhTx1, PhTx2, PhTx3, and PhTx 4 were identified. PhTx1, PhTx2, and PhTx3 are active on mammals and differ in their lethality and effects in mice (Rezende et al., 1991). PhTx4 produces marked stimulatory effects in insects and is more toxic to insects than to mammals (Figueiredo et al., 1995). More recently, it was shown that some toxins from the PhTx4 fraction, besides acting on insect sodium channels (de Lima et al., 2002), also can act on mammal sodium channels (Paiva et al., 2016; Silva et al., 2015a), although no apparent toxicity was observed when injecting (i.c.) these molecules in rat brain. The fifth fraction (PhM), apparently not toxic to mammals, is active on smooth muscle, causing contraction (Rezende et al., 1991).The average LD50 by i.c. injection in mice for the whole venom, PhTx1, PhTx2, PhTx3, and PhTx4, was 47, 45, 1.7, 137, and $480 \mu \mathrm{g} / \mathrm{kg}$, respectively (Rezende et al., 1991). PhTx2 is the fraction which displayed the strongest neurotoxic activity while PhTx5 or PhM ( $15 \mathrm{mg} / \mathrm{kg}$, i.v. injection) has no lethal effect in mice (Rezende et al., 1991). Fig. 2 shows a flow diagram describing the purification of Phoneutria nigriventer venom fractions.

\section{PhTx1}

The fraction PhTx1 is constituted by just one peptide, initially called Toxin 1 or Tx1. Afterwards, it was given the name Phoneutria nigriventer Toxin 1 or PnTx 1 . This peptide represents $0.45 \%$ of the whole venom protein content and it was the first purified and sequenced neurotoxin from $P$. nigriventer venom (Diniz et al., 1990). PnTx1 has a molecular mass of 8594.6 Da and is constituted of 78 amino acid residues, 14 of which are cysteines (Fig. 3). It is thus a fairly large peptide with a complex disulfide bridge pattern that remains undetermined till today.

Early research indicated that PnTx1 induces excitation and spastic paralysis in mice upon i.c. injection (Rezende et al., 1991). PnTx1 is lethal to rodents and has an astonishing $\mathrm{LD}_{50}$ of $5.5 \mathrm{pmol} / \mathrm{g}$ in mice (Diniz et al., 1993, 2006; Klint et al., 2012). Nevertheless, the exact molecular target of this toxin remained unknown for many years. Initially, it was suggested that PnTx1 could act on calcium channels because radio-iodinated ${ }^{125} \mathrm{I}$-PnTx 1 showed partial competition with the 


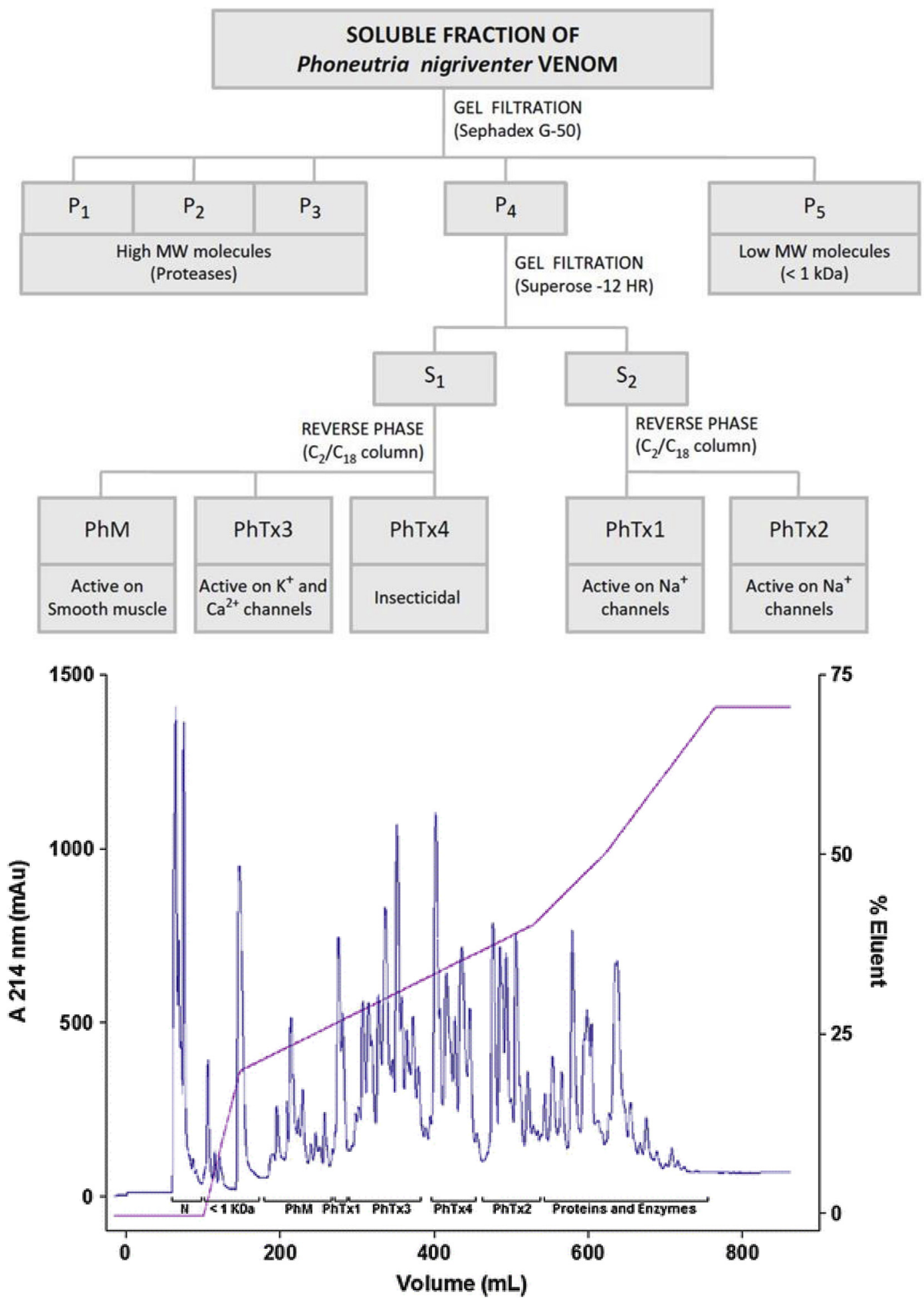

Fig. 2. Upper panel: Flowchart showing the purification procedure of Phoneutria nigriventer venom fractions. Adapted from de Lima et al., 2016). Lower panel: Elution profile of reversed-phase (RP-HPLC) fractionation of Phoneutria nigriventer venom. Venom sample was loaded on a preparative Vydac C4 column $(2.2 \times 25 \mathrm{~cm})$. Column was eluted at a flow rate of $5 \mathrm{~mL} / \mathrm{min}$, monitored at $214 \mathrm{~nm}$, under a gradient of acetonitrile (Richardson et al., 2006). The solid bars indicate the eluted fractions and (N) nigriventrine. Figure adapted from de Lima et al., 2016.

\section{0 \\ 20 \\ 30 \\ 40 \\ 50 \\ 60 \\ 70 \\ AELTSCFPVGHECDGDASNCNCCGDDVYCGCGWGRWNCKCKVADQSYAYGICKDKVNCPNRHLWPAKVCKKPCRRNCGG}

Fig. 3. Sequence of PnTx1. Cysteines are indicated in red. (For interpretation of the references to colour in this figure legend, the reader is referred to the Web version of this article.) 
fraction PhTx3, which contains several calcium channel modulating peptides. However, labeled PnTx1 did not compete with $\omega$-conotoxin GVIA, a calcium channel inhibiting toxin isolated from cone snail venom (Santos et al., 1999). This rather contrary result was later attributed to the presence of a small contamination with PnTx3-3. PnTx33 is a well-characterized toxin that potently inhibits high-voltage-activated Cav channels, but not low-voltage-activated Cav channels (Leao et al., 2000). This contamination could explain the partial competition for Cav channel inhibition (Martin-Moutot et al., 2006). However, the question whether or not PnTx1 interacts with Cav channels can still not be answered unambiguously. Experiments using a highly purified PnTx1 showed that this toxin partially displaced the calcium-antagonist dihydropyridine derivative 3H-PN200-110 in GH3 cell membranes. Furthermore, a 50\% inhibition of the calcium influx in GH3 cells was observed after application of $1 \mu \mathrm{M}$ PnTx1 (Santos et al., 1999). On the other hand, experiments with the recombinant PnTx1 (rPnTx1) showed no modification in the calcium currents of dorsal root ganglia (DRG) neurons (Silva et al., 2012). One explanation for these seeming contradictorily observations could be that PnTx1 does interact with Cav channels but only selectively with a certain specific subtype of Cav channels. Nonetheless, PnTx1 awaits further investigation in order to elucidate its Cav channel interaction.

Using similar competitive binding experiments it was shown that ${ }^{125}$ I-PnTx1 partially blocked the PhTx2 induced activity in myenteric plexus-longitudinal muscle preparations (Santos et al., 1999). Since PhTX2 is the fraction that contains mainly sodium channel toxins, this observation hinted towards an activity of PnTx1 on Nav channels. Interestingly, ${ }^{125}$ I-PnTx1 did not compete with PnTx2-6, a toxin that modulates the inactivation process of Nav channels (Silva et al., 2015a). The authors consequently concluded that PnTx1 might be interacting with Nav channels via another binding site on the Nav channel (Santos et al., 1999). Later on, these conclusions were shown to be correct when it was demonstrated that PnTx1 competes with $\mu$-conotoxin GIIIA (Martin-Moutot et al., 2006). $\mu$-conotoxins are small peptides isolated from cone snail venom that inhibit Nav channels with great potency and selectivity (Green et al., 2014; Green and Olivera, 2016; Prashanth et al., 2014). They are known to bind a micro site within the neurotoxin binding site 1 of the Nav channel (French et al., 2010). It is interesting to note that PnTx1 competes with $\mu$-conotoxins for site 1 but not with the small compound Nav channel inhibitor tetrodotoxin (TTX). Using the patch-clamp technique and Chinese hamster ovary cells expressing Nav1.2 channels, PnTx1 was characterized as a state-dependent channel inhibitor, preferring to interact with the Nav channels in the open state (Martin-Moutot et al., 2006).

The recombinant PnTx1 was expressed in a bacterial heterologous system and inhibited a variety of sodium channel isoforms expressed in Xenopus laevis oocytes (Fig. 4) and native sodium channels in DRG neurons (Silva et al., 2012). Surprisingly, recombinant toxin displayed a 3 -fold lower $\mathrm{IC}_{50}$ value compared to native peptide. This could possibly be explained by the presence of three additional amino acids in the sequence of the recombinant produced toxin, namely alanine and methionine at the N-terminus and a glycine at the C-terminus. The recombinant toxin, $\mathrm{PPnTx} 1$, inhibited mammalian Nav channel isoforms with the following order of potency: rNav1.2 > rNav1.7 rNav1.4 rNav1.3 > mNav1.6 hNav1.8 with no effect on Nav1.5 (Silva et al., 2012). Accordingly, rPnTx1 was less effective on TTX-resistant sodium channels in DRG neurons (Silva et al., 2012). From a drug development point of view, it is interesting to note that PnTx1 does not inhibit the cardiac isoform Nav1.5. Indeed, such a significant selectivity towards neuronal sodium channels is considered appealing as this avoids unwanted cardiovascular side effects. Inhibition of Nav channels occurred without modification of the biophysical properties of the channels since no alterations of the voltage dependence of activation and steady-state inactivation were noted (Martin-Moutot et al., 2006; Silva et al., 2012). Competitive binding studies have shown that PnTx1 does not compete with toxins acting on site $2,3,4,5$. Nor does PnTx 1 bind to the interaction site for local anesthetics or for pyrethroids (Martin-Moutot et al., 2006). Furthermore, using mutated Nav1.2 channels devoid of fast inactivation, it was shown that PnTx1 does not stabilize the channels in the inactivated state (Silva et al., 2012). It thus can be concluded that the PnTx1 induced sodium current inhibition is not a result of modified gating and open time probability but rather from a physical obstruction of the sodium ion pathway through the Nav channel. The result of PnTx1 binding is thus a reduction of the single channel conductance.

PnTx1 does not inhibit insect Nav channels. Even at higher concentrations, PnTx1 failed to decrease the current peak amplitude of arthropod Nav channel isoforms such as from the fruit fly Drosophila melanogaster (DmNav1), the cockroach Blattella germanica (BgNav1.1) and the mite Varroa destructor (VdNav1). This inactivity on arthropod Nav channels corroborates well with previous studies showing a lack of insecticidal activity of venom fraction PhTx1, when injected in insects (Santos et al., 1999).

Curiously, both native and recombinant toxins were not able to block $100 \%$ of the Nav1.2 currents, reaching a maximal inhibition of approximately $85 \%$ of the sodium peak current, even at saturating conditions. Subsequent addition of TTX results in completely inhibited sodium channels indicating that TTX can still reach and bind its site of interaction, independent of the presence of PnTx1 (Silva et al., 2012). Since these experiments were performed in Xenopus oocytes heterologously expressing Nav channels it was concluded that PnTx1 incompletely inhibits the sodium conductance through the channel, a phenomenon shared with the $\mu$-conotoxins (French et al., 2010; Silva et al., 2012).

Moreover, an interesting sequence comparison can be made between a central segment of PnTx1 and $\mu$-conotoxins such as GIIIA and KIIIA. In this way, the amino acids W33, R35 and K39 of PnTx1 can be aligned with the similar amino acids W8, R10 and R14, present in the $\mu$ conotoxin KIIIA (Khoo et al., 2011; Silva et al., 2012; Zhang et al., 2010). $\mu$-conotoxins have been intensively studied and for several of them are the key residues determined (Prashanth et al., 2014; Zhang et al., 2010). Single mutations can alter significantly the selectivity of a toxin. The substitution of tryptophan at position 8 by arginine decreased affinity of $\mu$-conotoxin KIIIA for Nav1.2 subtype, making it more selective to Nav1.4 (Van Der Haegen et al., 2011). There are three basic amino acids conserved in $\mu$-conotoxin GIIIA that are putative key residues for the interaction with Nav channels: R13, K16, and K19. PnTx1 has basic residues in two correspondent positions, R35 (instead K16) and K39 (corresponding to K19). However, PnTx1 lacks the first arginine (R13) and has a glycine (G32) in the corresponding position. Arginine-13 was postulated to be a general residue for $\mu$-conotoxins to interact with the receptor site of sodium channels. This residue is particularly critical, since it is believed to compete with the guanidinium group of TTX or STX for the binding site 1. The toxin binding sites of sodium channels were classified based on their ability to compete with other toxins in binding experiments. Site 1 is the binding site of TTX and STX and toxins that can displace them, such as $\mu$-conotoxin GIIIA. Since PnTx1 competes with $\mu$-conotoxin GIIIA but not with TTX, it would be more appropriate to consider it as a macro site 1 instead (de Lima et al., 2016).

Based on all information to date, one hypothesis is a mechanism of action where PnTx1 binds to the outer mouth of the channel pore, at a site similar or at least partially overlapping with the $\mu$-conotoxin binding site within neurotoxin binding site 1 . Upon binding, PnTx1 hinders the flow of sodium ions. Presumably, the inhibition is a result of both physical occlusion and repulsion of positive charges, similar to what has been proposed for the $\mu$-conotoxins (French et al., 2010). Therefore, the likely mechanism of action of PnTx1 would be the reduction of the unitary conductance of the channel, similarly to what is seen for the mutated toxin $\mu$-conotoxin GIIIA (R13Q) (Becker et al., 1992).

Spider venom peptides targeting Nav channels (NaSpTxs) have been 

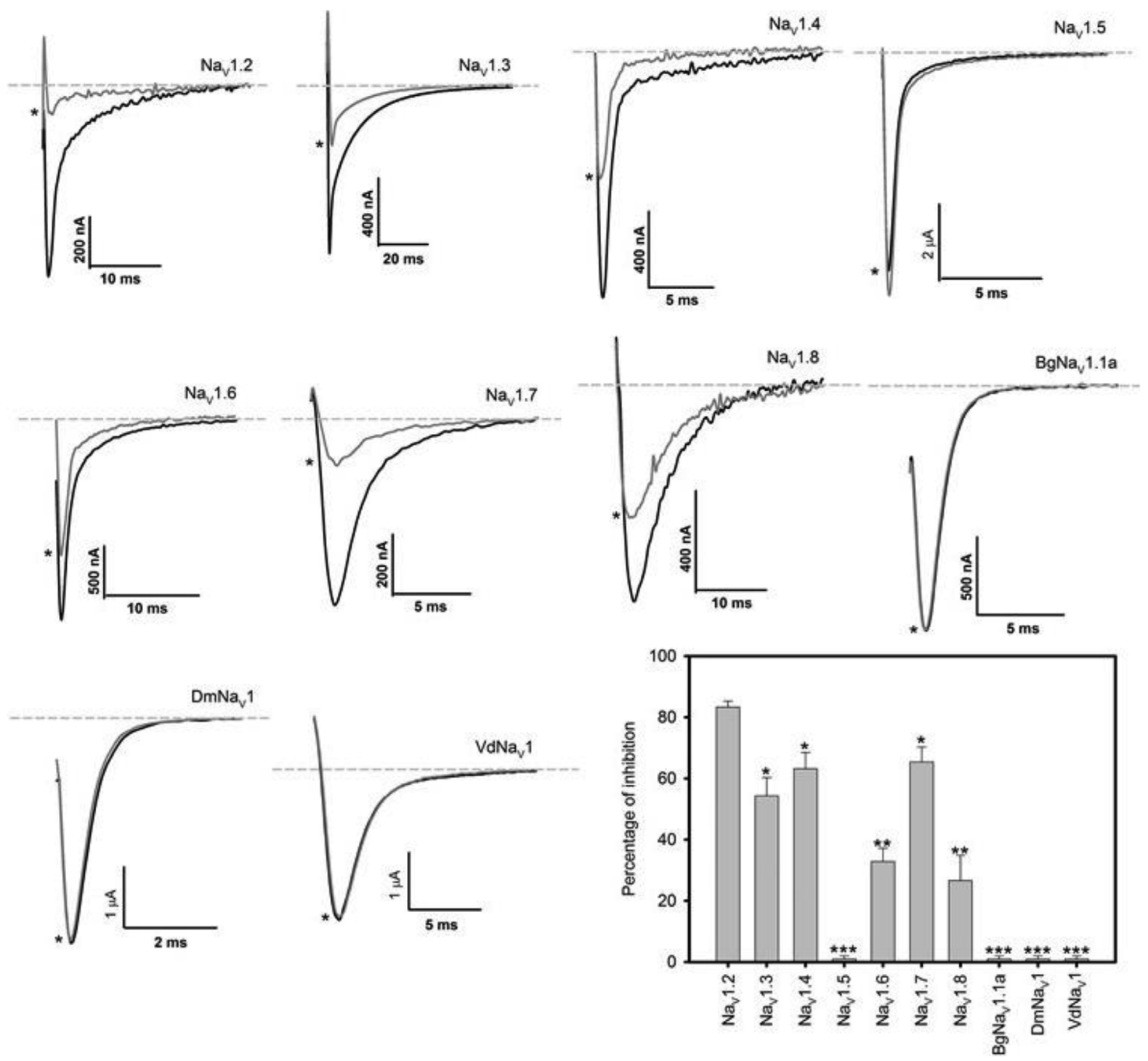

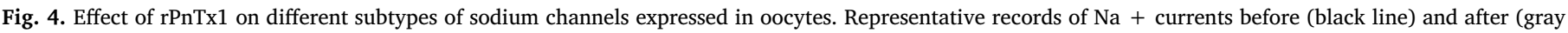

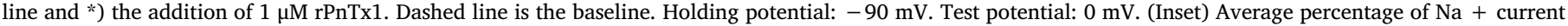

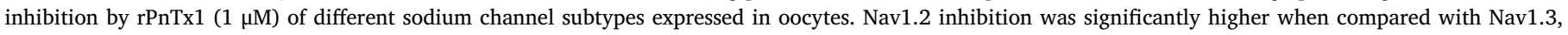

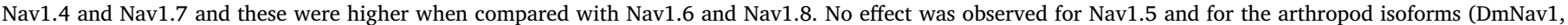

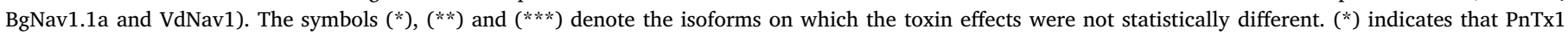

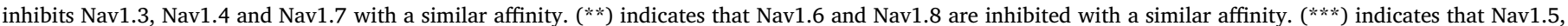
DmNav1, BgNav1 and VdNav1 are not inhibited by PnTx1. Figure adapted from Silva et al. (2012).

divided into 12 families based on sequence identity and intercysteine spacing (Klint et al., 2012). PnTx1 is the representative of family 8 which constitutes the family of the longest NaSpTxs. So far, no structure, or even disulfide bridge pattern for that matter, has been determined for this structural family. Moreover, none of the Phoneutria nigriventer peptides has its structure determined so far, emphasizing the challenges, and herewith the opportunities from a structural point of view, to be found in the Phoneutria venom. This is mainly due to peptide scarcity since only small amounts can be isolated from the venom and due to the peptide size and complexity. Therefore, many structurefunction questions about PnTx1 remain open, which makes it an attractive object for future studies. Elucidation of the structure of PnTx1 would greatly enhance the understanding on how this toxin exerts its interesting pharmacology.

\section{PhTx2}

The fraction PhTx2 was found to be toxic to both mice and insects (Figueiredo et al., 1995). Intracerebral injection in mice resulted in priapism, salivation, convulsions and spastic paralysis of the anterior and posterior limbs among other manifestations (Rezende et al., 1991). Using a frog skeletal muscle preparation, it was shown that fraction PhTx2 alters the Nav channel kinetics. PhTx2 caused a shift in the activation and steady-state inactivation curves, a slowing down of the channel inactivation and a partial inhibition of the sodium peak current. The same experiments indicated that PhTx2 does not affect the potassium current (Araujo et al., 1993). Phoneutria.

Purification of fraction PhTx2 resulted in 9 peptides which were named PnTx2-1 till PnTx2-9 (Cordeiro et al., 1992). All peptides were investigated for their toxicity by i.c. injection in mice. PnTx2-2, PnTx23, PnTx2-4, PnTx2-7, and PnTx2-8 showed low toxicity and these peptides still await justification for their classification as neurotoxin. 


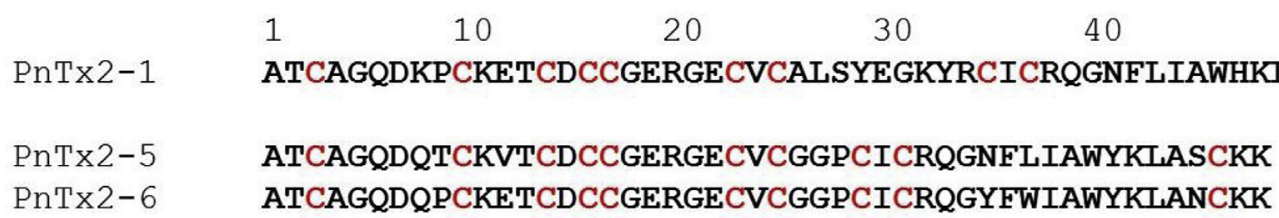

PnTx2-9 SFCIPFKPCKSDENCCKKFKCKTTGIVKLCRW

Fig. 5. Sequences of the toxins identified in Phoneutria nigriventer venom fraction 2. Cysteines are indicated in red. (For interpretation of the references to colour in this figure legend, the reader is referred to the Web version of this article.)

PnTx2-1, PnTx2-5, and PnTx2-6 share up to 77\% identity with each other (Fig. 5) and all 3 toxins elicit neurotoxic effects when injected into mice (Table 1) (Cordeiro et al., 1992). Pruritus, lacrimation, increased salivation, sweating, and agitation followed by spastic paralysis of the limbs was observed. PnTx2-9, was much less toxic to mice, causing pruritus, reduction in motility and tail erection. PnTx2-9 differs in sequence from the other peptides of PhTx2. It is a rather short peptide of 32 residues with 3 disulfide bridges. Although annotated as a Nav channel toxin, no functional data is available to justify this classification.

PnTx2-1 remains scarcely studied. The in vivo tests in mice and based on the sequence homology with PnTx2-5 and PnTx2-6, it can be assumed that this peptide also targets Nav channels. However, functional studies are required to investigate the subtype selectivity and species specificity of PnTx2-1 (Richardson et al., 2006).

Among all the polypeptides purified from PhTx2 fraction, PnTx2-5 and PnTx2-6 are the most studied toxins. They have high sequence homology, differing only in five amino acid residues (Cordeiro et al., 1992). Both toxins were shown to modulate sodium channel kinetics by slowing down the inactivation process and by shifting the voltage dependence of activation towards more hyperpolarized potentials. Both peptides were found to induce a painful and persistent penile erection, also known as priapism which is a common clinical manifestation upon Phoneutria nigriventer envenomation. Interestingly, despite the high homology between both peptides, significant differences in potency have been reported. PnTx2-5 displayed an approximately 6-fold lower potency than PnTx2-6 in electrophysiological assays (Matavel et al., 2002, 2009; Nunes et al., 2010).

Using frog skeletal muscle, an in-depth characterization of PnTx2-6 on a molecular level was performed. PnTx2-6 induces a plethora of modifications of channel kinetics. PnTx2-6 reduces the sodium peak current, slows down the time constant for fast inactivation and causes a hyperpolarizing shift of the voltage dependence of both the activation and steady-state inactivation curves (Matavel et al., 2002). The diversity of channel modulations is intriguing from a biophysical point of view. The slowing down of the inactivation process is similar to the activity of certain scorpion, sea anemone and other spider toxins such as the $\beta / \delta$-agatoxins (Cardoso and Lewis, 2017; Deuis et al., 2017; Israel et al., 2017; Nicholson, 2007; Wanke et al., 2009). The subtype selectivity for mammalian Nav channel isoforms was determined for PnTx2-6 (Fig. 6) (Silva et al., 2015a). These toxins have been characterized to bind to the so called neurotoxin binding site 3 Nav channels (Catterall et al., 2007). These site 3 toxins slow down sodium channel inactivation and in many cases they induce other channel gating modifications as well (Cardoso and Lewis, 2017; Peigneur et al., 2012; Zhu et al., 2012). Binding experiments in brain synaptosomes showed that PnTx2-6 partially competes with the typical $\alpha$-scorpion toxin AaHII (from Androctonus australis Hector) (Matavel et al., 2009). A shift of the midpoint of activation potential toward more negative potentials together with a reduction in sodium peak current are characteristics of gating alterations often observed for $\beta$-scorpion toxins and certain spider toxins (Leipold et al., 2006; Nicholson et al., 2004; Peigneur et al., 2015). However, it was shown that PnTx2-6 does not compete with the $\beta$-scorpion toxin CssIV (from Centruroides suffusus suffusus) and it thus seems unlikely that PnTx2-6 binds to same site as $\beta$-scorpion toxin (Matavel et al., 2009). As such, structure-function studies are required to pinpoint which toxin-channel interactions are responsible for the PnTx2-6 induced channel modulations.

The effect of PnTx2-6 on spontaneous penile erection is remarkable and opens perspectives for clinical applications. In a first step to further investigate the exact role of PnTx2-6 in erectile function, the toxin was expressed recombinantly in E. coli cells (Torres et al., 2010). The recombinant toxin was able to produce erection, similar to native toxin (Torres et al., 2010). The mechanism of action through which PnTx2-5 and PnTx2-6 promote cavernosal relaxation and enhance erectile function is not completely clarified (Nunes et al., 2010, 2012a; Yonamine et al., 2004). However, intensive research in recent years has provided a better understanding on how these toxins influence erectile function (Nunes et al., 2012b). It is suggested that PnTx2-6 intervenes in the nitric oxide (NO)/cyclic GMP pathway by increasing the release of NO in the corpus cavernosum tissue (Nunes et al., 2008). The release of NO from penile endothelial cells or nitrergic nerves is a key regulator in erectile function. Increased NO concentrations, triggered by sexual stimulation, induces relaxation of penile smooth muscle. This relaxation results in an increased blood flow and intracavernosal pressure which leads to penile erection. The PnTx2-6 induced relaxation is neuronal nitric oxide synthase (nNOS) depended (Nunes et al., 2012b). The activation of nNOS by PnTx2-6 is most likely indirect. Since PnTx2-6 is characterized as a modulator of Nav channel inactivation, the binding of PnTx2-6 to Nav channels will result in an increased sodium influx. This will trigger a cascade of cellular reactions, eventually resulting in an activation of nNOS and consequently stimulation of NO release. This mechanism of action was supported by the observation that $\omega$-conotoxin GVIA, an inhibitor of N-type calcium channels, could block the relaxation induced by PnTx2-6. Additionally, it was shown that the cavernosal relaxation provoked by PnTx2-6 is not dependent on phosphodiesterase-5 (PDE5) inhibition (Nunes et al., 2012b). Furthermore, it was shown that PnTx2-6 induces priapism in mice even after cavernosal denervation, indicating that the toxin might not depend on cavernosal nerves integrity (Ravelli et al., 2017). Investigating the gene expression in mice erectile tissue showed overexpressing of two genes potentially related to PnTx2-6 induced priapism (Villanova et al., 2009). One of these genes is directly involved in the activation of the NO/cGMP pathway.

PnTx2-5 has been less investigated, compared to PnTx2-6, but the results that are available do suggest that this toxin is involved in penile potentiation similar to PnTx2-6 (Villanova et al., 2009). PnTx2-5 also caused penile erection when injected intraperitoneal in male mice. These effects are completely abolished by the nNOS-selective inhibitor 7-nitroindazole, indicating an important involvement of nNOS in this effect as well (Yonamine et al., 2004). Both toxins, PhTx2-5 and PhTx26 , represent interesting pharmacological tools to study erectile dysfunction. A 19 residues peptide, devoid of disulfide bridges but comprising the pharmacophore of PnTx2-6 was designed. This PnTx2-6derived peptide, named PnPP-19, was no longer active on Nav channels. The abolishment of Nav channel activity can be considered as an 

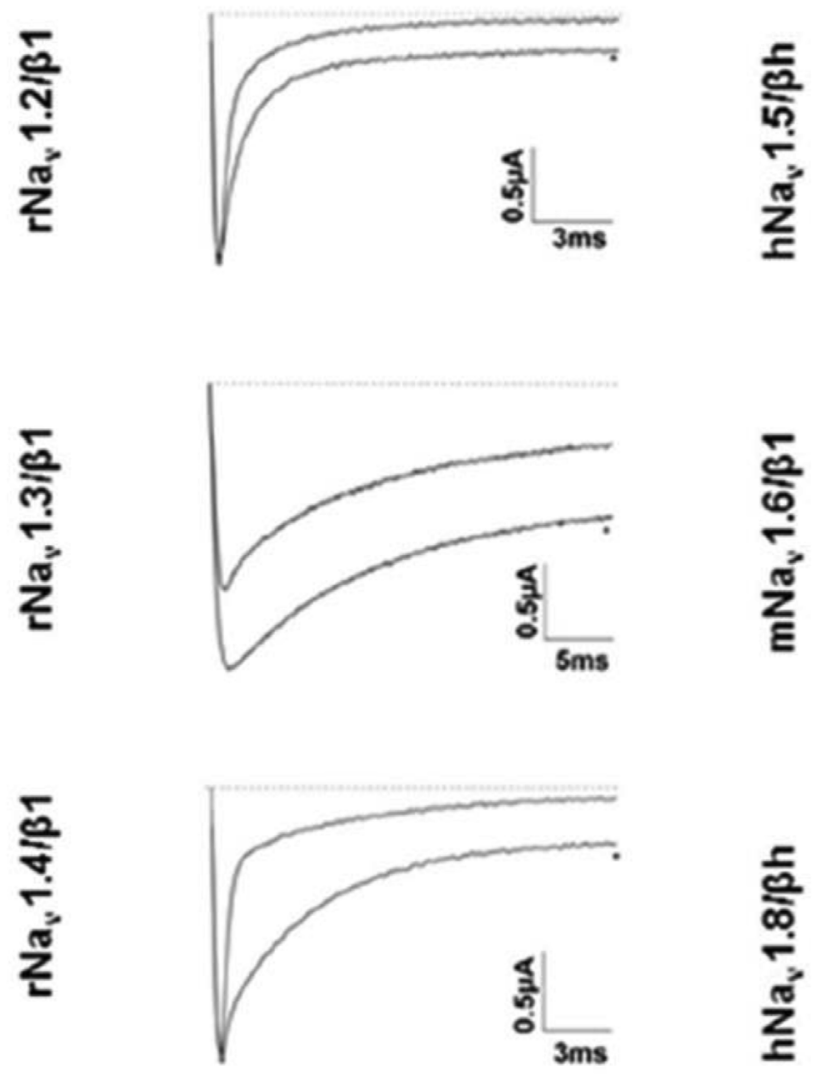
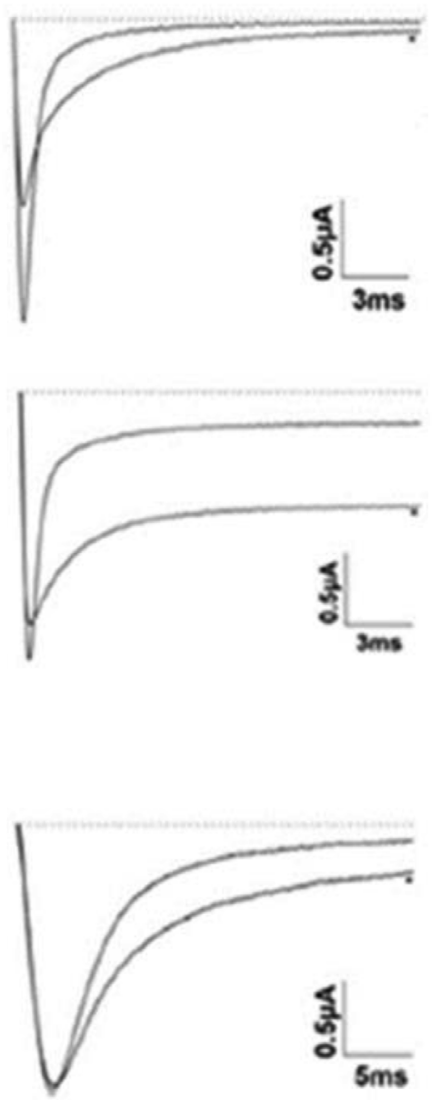

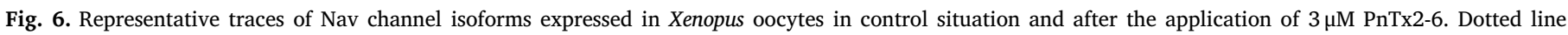
indicates zero current level. Asterisk indicates steady state current traces after toxin application. Figure adapted from Silva et al. (2015a,b).

important amenity since this strongly reduces the peptide toxicity and hereby unwanted side effects. Interestingly however, PnPP-19 potentiates penile erection with a similar activity as PnTx2-6 (Silva et al., 2015a). Therefore, PnPP-19 can be considered as an exciting lead compound for drug development related to the treatment of erectile dysfunction. Moreover, PnPP-19 has also been investigated for its role in both peripheral and central antinociception (Freitas et al., 2016). It was shown that PnPP-19 inhibits neutral endopeptidase and activates receptors involved in pain pathways such as the cannabinoid receptor 1 and the $\mu$ - and $\delta$-opioid receptors (da Fonseca Pacheco et al., 2016; Freitas et al., 2016). Furthermore, the peripheral antinociceptive effect induced by PnPP-19 is resulting from an activation of the NO-cGMP$\mathrm{K}_{\mathrm{ATP}}$ pathway. Hereby, an activation of both endothelial nitric oxide synthase (eNOS) and nNOS by PnPP-19 occurs in rat paw Freitas et al. (2017). PnPP-19 selectively activates $\mu$-opioid receptors inducing indirectly inhibition of calcium channels and hereby impairing calcium influx in dorsal root ganglion (DRG) neurons. Interestingly, notwithstanding the activation of opioid receptors, PnPP-19 does not induce $\beta$ arrestin2 recruitment. PnPP-19 is the first spider toxin derivative that, among opioid receptors, selectively activates $\mu$-opioid receptors. The observed lack of $\beta$-arrestin2 recruitment highlights the potential of this peptide for the design of new improved opioid agonists (Freitas et al., 2018). One of the very serious and life-threatening conditions developed following the use of the usual opioid agonist medicines is respiratory paralysis. It has been demonstrated that the induction of respiratory paralysis, as well as other side effects, after the use of opioids may be linked with the recruitment of the $\beta$-arrestin pathway, which is stimulated downstream following activation of $\mu$-opioid receptor. Since opioid receptors are still one of the most relevant targets for pain treatment, great effort is being put in the development of new opioid agonists that elicit fewer negative side effects. In this way, the lack of $\beta$ - arrestin2 recruitment by PnPP-19 underlines the potential of this peptide as a possible lead compound in the development of improved opioid agonists (Freitas et al., 2018).

\section{PhTx3}

Injection in mice of fraction PhTx3 results in a progressive flaccid paralysis of all legs (Kushmerick et al., 1999; Prado et al., 1996; Rezende et al., 1991). Of all venom fractions, PhTx3 must be the most studied and best characterized fraction. This is a consequence of the pharmacological potential present in this fraction. All of the PhTx3 peptides were found to target voltage-gated calcium (Cav) channels or Kv channels (Table 1). It is remarkable that up to date only one voltagegated potassium channel targeting peptide (KSpTxs) has been characterized from Phoneutria venom while other spiders are known to produce potent and pharmacological interesting KSpTxs (Priest et al., 2007; Swartz, 2007). The genes encoding for all PhTx3 toxins are identified. It was shown that these toxins are encoded as a precursor peptide composed of a signal peptide, an intervening propeptide, and the mature toxin (Cardoso et al., 2003; Carneiro et al., 2003; Kalapothakis et al., 1998b). Interestingly, certain isoforms identified by molecular cloning have never been purified from the crude venom. However, some of these peptides were functionally expressed in heterologous systems in order to allow functional characterization (Carneiro et al., 2003; Souza et al., 2008). Many studies have been devoted to the identification of the molecular target of PhTx3. First studies showed that this venom fraction reduces the release of tritiated acetylcholine in the brain indicating a potential target involved in the acetylcholine release in the brain and in the autonomic nervous system. Therefore, the target could be a calcium channel (de Lima et al., 2016; Gomez et al., 1995). This hypothesis was further supported by the 


\section{$1 \quad 10 \quad 20 \quad 30 \quad 40$ \\ PnTX3-1 AECAAVYERCGKGYKRCCEERPCKCNIVMDNCTCKKFISEL \\ 50 \\ 60 \\ 70 \\ PnTX3-2 -ACAGLYKKCGKGASPCCEDRPCKCDLAMGNCICKKKFIEFFGGGK \\ PnTX 3 -3 GCANAYKSCNGPHTCCWGYNGYKKACICSGXNWK \\ PnTX 3-4 SCINVGDFCDGKKDDCQCCRDNAFCSCSVIFGYKTNCRCEVGTTATSYGICMAKHKCGRQTTCTKPCLSKRCKKNHG}

PnTX3-5 GCIGRNESCKFDRHGCCWPWSCSCWNKEGQPESDVWCECSLKIGK

\section{PnTX3-6 ACIPRGEICTDDCECCGCDNQCYCPPGSSLGIFKC SCAHANKYFCNRKKEKCKK}

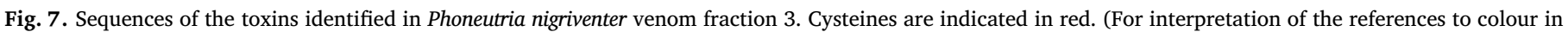
this figure legend, the reader is referred to the Web version of this article.)

observation that PhTx3 abolishes calcium-dependent glutamate release in rat brain cortical synaptosomes without influencing the calcium-independent exocytosis. PhTx3 partially inhibited the glutamate release without affecting the glutamate release triggered by intracellular calcium stocks. This hinted towards the presence of toxins in $\mathrm{PhTx} 3$ which interfere with the calcium influx in synaptosomes (Prado et al., 1996).

From this fraction six toxins, named PnTx3-1 to PnTx3-6, have been characterized (Fig. 7). PhTx3 is the venom fraction composed of the most heterogeneous group of toxins. The peptides found in PhTx3 share little sequence identity, and therefore, display a wide array of differing pharmacological activities when injected in vivo. For example, PnTx3-1, PnTx3-5, and PnTx3-6 induce paralysis of the posterior limbs. PnTx3-2 induces immediate clockwise gyration and flaccid paralysis. PnTx3-3 and PnTx3-4 are the most toxic: at $5 \mu \mathrm{g} /$ mouse they reproduce the fast flaccid paralysis followed by death observed for the whole PhTx3 fraction (Cordeiro et al., 1993; de Lima et al., 2016). PnTx3-6 also induces analgesia models pain in rodents (Souza et al., 2008).

It was shown that PnTx3-1 increases calcium oscillation in GH3 cells, presumably by blocking potassium currents (Kushmerick et al., 1999). Whole-cell patch clamp experiments showed that PnTx3-1 reversibly and selectively inhibits type-A potassium current (IA) without affecting the delayed or the inward rectifying potassium current. PnTx3-1 has no effect on large conductance calcium sensitive potassium channels. Furthermore, this toxin does not interact with T- and L-type Cav channels. The inhibition of IA favors cell depolarization and Cav channel activation, increasing the frequency of calcium oscillation (Kushmerick et al., 1999). It is important to note that these patch clamp experiments in GH3 cells do not rule out that the observed effects on oscillation frequency are following upon interaction of PnTx3-1 with an unknown target. Further research is required to verify that Tx3-1 is indeed a potassium channel toxin and to determine which potassium channel isoforms this peptide is targeting. In the heart, PnTx3-1 had an anti-arrhythmogenic effect, decreasing the ACh-mediated heart rate by doubling the frequency of spontaneous miniature end plate potential protecting ischemia/reperfusion heart against arrhythmia (Almeida et al., 2011; de Lima et al., 2016). More recently, it was shown that PnTx3-1 causes an antinociception by interfering with the choline esterase activating cholinergic system (Rigo et al., 2017).

PnTx3-2 is characterized as a selective inhibitor of L-type Cav channels (Kalapothakis et al., 1998a). PnTx3-2 does not show affinity for $\mathrm{N}$ - or $\mathrm{P} / \mathrm{Q}$-type Cav channels since the presence of this toxin did not modify the KCl-evoked glutamate release nor the rise of intracellular calcium in synaptosomes (Prado et al., 1996).

PnTx3-3 is considered as the most potent toxin from the venom fraction PhTx3. Many of the toxic properties of fraction PhTx3 are produced by this toxin (Guatimosim et al., 1997; Prado et al., 1996). PnTx3-3 inhibits P/Q- and R-type Cav channels with high affinity. At higher concentrations, PnTx3-3 also inhibits L- and N-type Cav channels (Leao et al., 2000). Interestingly, PnTx3-3 induces a different profile of behavioral effects compared to the well characterized $\omega$ conotoxin MVIIC which is also an inhibitor of P/Q-type Cav channels.
Ample $\omega$-conotoxins have been identified from cone snail venom (Prashanth et al., 2014). Noteworthy, all studies with $\mathrm{PhTx} 3$ peptides acting on Cav channels have used either GVIA or MVIIC $\omega$-conotoxins to compare the analgesic activity. A more recent study investigated the effects of PnTx3-3 on sensory transmission in the spinal cord of rats (Dalmolin et al., 2017). Using in vivo electrophysiological recordings, it was shown that PnTx3-3 exerts a prevalent antinociceptive effect mainly by inhibiting R-type Cav channels (Dalmolin et al., 2017).

PnTx3-4 affects the neurotransmission by blocking presynaptic calcium channels associated with exocytosis in mammals, lower vertebrates and arthropods (de Lima et al., 2016; Troncone et al., 2003). PnTx3-4 potently inhibits high-voltage-activated Cav channels in the sensory neurons of dorsal root. No activity was observed on low-voltage-activated Cav channels (Cassola et al., 1998). Moreover, PnTx3-4 is suggested to act on P/Q-type Cav channels (Miranda et al., 2001). Using heterologous expressed channels in HEK293 cells, it was shown that PnTx3-4 produced a potent and almost irreversible inhibition of $\mathrm{P} /$ Q-type Cav2.1 channels and N-type Cav2.2. Only a partial and reversible inhibition of R-type Cav2.3 channels was observed (Dos Santos et al., 2002). Furthermore, this toxin blocked potassium-induced and capsaicin-induced glutamate release from rat brain synaptosomes (Goncaves et al., 2011; Reis et al., 1999). Incubation of synaptosomes with PnTx3-4 in the presence of the calcium chelator EGTA blocked calcium-independent glutamate release, contrasting with the observation that the fraction PhTx3 did not inhibit calcium-independent components of glutamate release (de Lima et al., 2016). This seemingly contradiction could be explained by the low amount of PnTx3-4 present in the venom fraction PhTx3 (de Lima et al., 2016). The fraction PhTx3 exhibits a strong neuroprotective effect against neuronal damage induced by oxygen deprivation and low glucose (ODLG) insults on hippocampal slices (Pinheiro et al., 2006). In an in vitro model of retinal ischemia induced by ODLG, the fraction $\mathrm{PhTx} 3$ protected approximately $80 \%$ of the cells from injury (Agostini et al., 2011). PnTx3-3 and PnTx3-4 were identified as the peptides responsible for this neuroprotective effect. Both toxins were effective in preventing cell death after ischemic injury. It has been shown that ischemic injury induced by ODLG insults in retinal slices leads to increased levels of glutamate. These increased concentrations of glutamate cause retinal cell death. PnTx3-3 and PnTx3-4 exert their neuroprotective effect by indirectly inhibiting the glutamate increase through the inhibition of $\mathrm{N}$ and $\mathrm{P} / \mathrm{Q}$ type Cav channels. PnTx3-4 showed superior protection when compared to PnTx3-3, the fraction PhTx3, or the Cav channel inhibiting cone snail toxins $\omega$-conotoxin GVIA and $\omega$-conotoxin MVIIC (Agostini et al., 2011; Binda et al., 2016; Pinheiro et al., 2009). A similar neuroprotective activity was observed for both toxins in an in vitro model of brain ischemia. By using electrophysiology and the glutamate release assay in hippocampal slices, it was observed that both PnTx3-3 and PnTx3-4 blocked the glutamate release (Pinheiro et al., 2009). Furthermore, the neuroprotective effect of PnTx3-4 was also evidenced in vivo using a rat model of NMDA-induced injury of the retina (Binda et al., 2016). These results underline the role of PnTx3-4 as a novel 


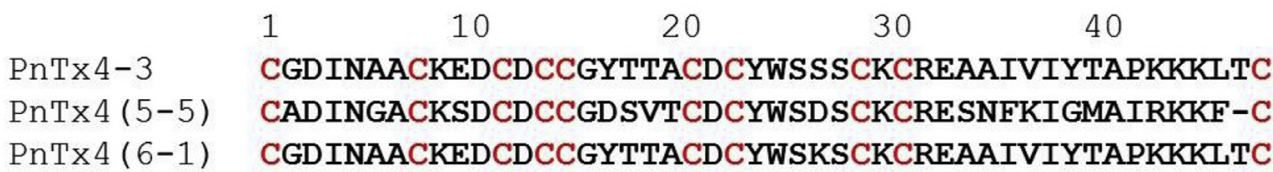

Fig. 8. Sequences of the toxins identified in Phoneutria nigriventer venom fraction 4. Cysteines are indicated in red. (For interpretation of the references to colour in this figure legend, the reader is referred to the Web version of this article.) potential tool to study or treat retinal injury. It is remarkable to note that the complete, correct sequence for PnTx3-4 matching the mass of the native toxin has still not been published despite the growing number of studies using this toxin (Herzig, 2016). As can be seen in Table 1, several PnTx3-4 isoforms have been reported.

The least studied toxin of the venom fraction PhTx3 is the toxin PnTx3-5. However, this peptide is known to potently block L-type Cav channels (Leao et al., 2000). PnTx3-5 demonstrated promising antinociceptive activity in clinically relevant pain models of postoperative, neuropathic and cancer-related pain (Oliveira et al., 2016). Interesting to note is that PnTx3-5 also displayed efficacy in opioid tolerant animals. Indeed, the clinical applicability potential of L-type, as well as $\mathrm{N}$ type, Cav channel inhibitors such as PnTx3-5, can be found in their apparent synergism with morphine. Although the exact mechanism is still under investigation, it is suggested that long-term use of morphine results in an increased expression of L- and N-type Cav channels (Verma et al., 2009). Therefore, inhibitors of L-type Cav channels can induce analgesia but also potentiate the morphine-induced analgesia. This could significantly reduce the consumption of morphine in patients with chronic pain (Oliveira et al., 2016).

The toxin PnTx3-6 (also known as Pha1b) binds to a broad range of high-voltage-activated Cav channels. It inhibits N-, R- and P/Q-type Cav channels with a comparable affinity. At higher concentrations, this toxin also blocks L-type Cav channels. However, PnTx3-6 has no activity on T-type Cav channels (Vieira et al., 2005). More recent, it was discovered that the analgesic activity of PnTx3-6 results, besides of the inhibition of Cav channels, from the interaction with TRPA1 receptors. PnTx3-6 is a potent TRPA1 antagonist while having no affinity for TRPV1 or TRPV4 receptors (Tonello et al., 2017). To overcome scarceness of the native PnTx3-6, a recombinant form of the peptide has been produced. This recombinant peptide, annotated CTK 01512-2, showed similar activity as the native PnTx3-6 (Tonello et al., 2017). PnTx3-6 inhibited potassium-induced calcium-dependent glutamate release by blocking voltage-gated calcium channels, but it was not able to modify the calcium-independent process (de Lima et al., 2016). PnTx3-6 has been investigated for its analgesic proprieties. Using both native and recombinant peptide, it was shown that PnTx3-6 was efficient for the treatment of persistent pathological pain mediated by either glutamate release or capsaicin-induced calcium influx, but not involving capsaicin receptor inhibition (Castro-Junior et al., 2013; Souza et al., 2008). The therapeutic potential of PnTx3-6 was evidenced by the observation that in preclinical models of chronic neuropathic pain, this toxin has a similar efficacy but a higher therapeutic index than the clinically used ziconotide (McGivern, 2007; Tonello et al., 2014). PnTx3-6 was effective in potentiating the analgesic effect of morphine in mice. Furthermore, the same study showed that this peptide reduced the hyperalgesia, tolerance, constipation and withdrawal syndrome induced by repeated morphine injection (Tonello et al., 2014). Similar, PnTx3-6 also potentiates the analgesic action of TRPV1 blockers, underlining the potential of Cav channel inhibitors as adjuvant analgesic agents (Palhares et al., 2017). Because of its action on N- and P/Q-type Cav channels, PnTx3-6 has also been used as a tool to study the involvement of these channels in bladder dysfunctions such as cystitis (Silva et al., 2015b). It should be noted that Cav channel inhibitors, such as the PnTx3 toxins, have not only a potential in antinociception. Because of the relevance of Cav channels in tumor progression, these peptides could also be useful in cancer related studies (Nicoletti et al., 2017).

\section{PhTx4}

PhTx4 is historically referred to as the insecticidal fraction. It earnt this reference based on its high toxicity and lethality toward insects while displaying a minor toxicity when injected in mice. This fraction causes hyperactivity such as cramps, quivering, jerking of the limbs, and violent trembling of the body and the legs, leading to muscle fatigue and therefore causing paralysis in insects (de Lima et al., 2016; Figueiredo et al., 1995). It is suggested that PhTx4 acts on the glutamatergic system of both insects and mammals. Three toxins have been characterized from the venom fraction PhTx4 (Fig. 8 and Table 1). They were named PnTx4 (6-1), PnTx4 (5-5), and PnTx4 (4-3) (de Figueiredo et al., 2001; Figueiredo et al., 1995; Oliveira et al., 2003). These 3 insecticidal toxins share high sequence identity and as such are believed to exhibit similar pharmacological properties.

PnTx4 (4-3) is not very well studied compared to the other toxins in this venom fraction. However, this toxin induces excitatory effects when injected in houseflies and cockroaches. Furthermore, it inhibits the glutamate uptake in rat brain synaptosomes (Oliveira et al., 2003).

PnTx4 (6-1) and PnTx4 (5-5) act on insect sodium channels (De Lima et al., 2007; de Lima et al., 2002). Despite their apparent lack of toxicity to mammals, they have been shown to inhibit glutamate uptake in the mammalian central nervous system and to modulate the inactivation process of mammalian Nav channels (Mafra et al., 1999; Oliveira et al., 2003; Paiva et al., 2016). It was evidenced that PnTx4 (5-5) is a reversible antagonist of NMDA ionotropic glutamate receptor in rat brain neurons (de Figueiredo et al., 2001). Since inhibition of NMDA receptors has been proposed as a therapeutic mechanism of neuroprotection, PnTx4 (5-5) has been investigated for its potential to promote neuronal survival by blocking NMDA receptors. Nevertheless, more research is required to determine the NMDA receptor subtype selectivity of PnTx4 (5-5) (Silva et al., 2016). The activity of PnTx4 (55) on insect and mammalian Nav channels was characterized in depth (Paiva et al., 2016). The subtype selectivity among mammalian Nav channels was determined using the Xenopus oocyte expression system and recombinant produced PnTx4 (5-5). Among mammalian Nav channels, the toxin caused a delay in the channel inactivation and a reduction of the sodium peak current. This reduction in peak current is the result of a toxin induced shift of the channel activation towards more depolarized membrane potentials. PnTx4 (5-5) has the most pronounced effect on insect Nav channels. Using the cockroach Nav channel BgNav1, it was illustrated that PnTx4 (5-5) has a devastating modulatory effect on these channels (Fig. 9). Upon binding, this toxin causes a complete inhibition of the inactivation of the channel and an increase of the sodium peak amplitude. However, contrary to what was observed for mammalian Nav channels, no shift in the midpoint of activation was noted for insect Nav channels (Fig. 9) (Paiva et al., 2016).

The most active toxin of this fraction is the anti-insect neurotoxin PnTx4 (6-1). A detailed mode of action of PnTx4 (6-1) and PnTx4 (5-5) has been acquired with the double-oil-gap method using axonal preparations of the cockroach Periplaneta americana (de Lima et al., 2002). Both toxins induced evoked action potential prolongation in axonal preparations. This effect was stronger after PnTx4 (6-1) administration than after PnTx4 (5-5). Tests performed in a voltage-clamp configuration showed that both PnTx4 (6-1) and PnTx4 (5-5) prolonged the axonal sodium current in a manner similar to toxins binding to neurotoxin site 3 of Nav channels. Similar tests in Xenopus oocytes indicated that PnTx4 (6-1) has no effect on Nav1.2 and Nav1.4 channels (de Lima 
A

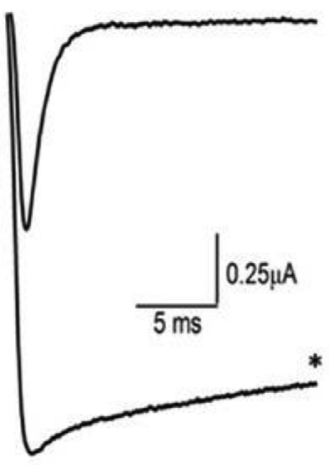

B

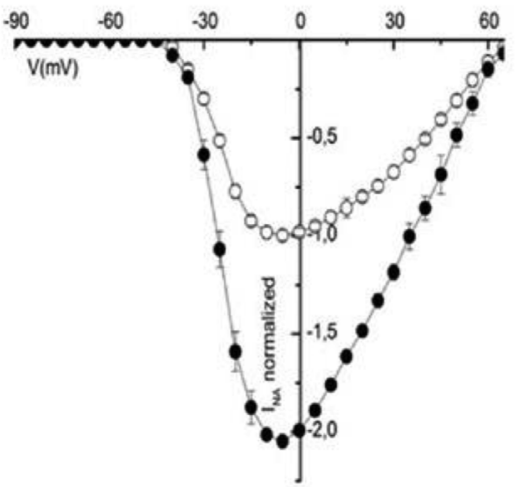

C

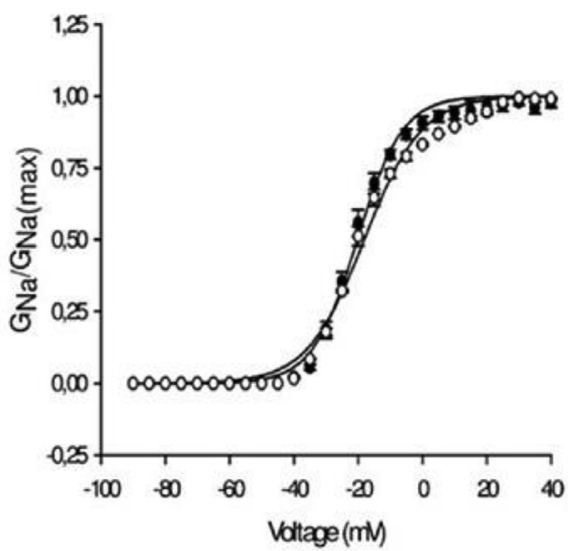

Fig. 9. Effects of PnTx4 (5-5) on the cockcroach B. germanica sodium channel (BgNav1) expressed in X. leavis oocytes. A) Representative whole-cell current traces in control and in presence of $1 \mu \mathrm{M} \mathrm{rPnTx} 4$ (5-5) (*). B) Current-voltage (I x V) relationships in control (O) and in presence of $1 \mu \mathrm{M} \mathrm{rPnTx} 4$ (5-5) (๑). C) Activation of the conductance in control $(O)$ and in presence of $1 \mu \mathrm{M} \operatorname{rPnTx} 4$ (5-5) (@), fitted with the Boltzmann equation. Figure adapted from Paiva et al. (2016).

et al., 2016; de Lima et al., 2002).

However, in DUM cells, PnTx4 (6-1) changed the regular spontaneous firing pattern of action potential generation into an irregular activity, evidencing its activity on insect Nav channels. The results obtained with electrophysiological experiments suggested that PnTx4 (6-1) is most likely binding to the neurotoxin binding site 3 of Nav channels. This was later on confirmed with binding assays using Bom IV, an $\alpha$-like scorpion toxin that binds to receptor site 3 on insect sodium channels. Bom IV was displaced by PnTx4 (6-1) (Figueiredo et al., 1995). More recently, it was found that PnTx4 (6-1) induces antinociception in inflammatory, neuropathic and acute pain models in rats. It is suggested that this antinociceptive effect involves the cannabinoid system, through cannabinoid receptor 1 , and the opioid system, through the $\mu$ - and $\delta$-opioid subtype receptors (Emerich et al., 2016).

\section{PhTx5}

The fifth Phoneutria venom fraction is also known as fraction $\mathrm{PhM}$ (Fig. 2, Table 2). An improved purification method of $P$. nigriventer venom combined with mass spectrometry analysis contributed for the identification of the fraction PhM (Pimenta et al., 2005; Richardson et al., 2006). This fraction was characterized for its activity on smooth muscle. However, the previous described purification method did not allow identification of these peptides because of their low levels in the whole venom and because their N-termini were somehow blocked which prevented sequencing by Edman degradation. It is also probable that some of these small molecules do not represent mature peptides, but are result of precursor processing and/or degradation of mature toxins (Pimenta et al., 2005). PhM consists of a pool of similar isoforms of smaller $(<2 \mathrm{kDa})$ peptides. The amino acid sequences of 15 of these isoforms (Fig. 10) were determined by mass spectrometry (Pimenta et al., 2005). These muscle-active peptides contain 7-14 amino acid residues and have a common scaffold composed of basic and acid amino acids (PyrKKDKKDx), where $\mathrm{x}$ can be either K or R. Since all of these molecules are structurally related to the tachykinin family of neurohormone peptides, which possess N-terminal pyroglutamate residues, they were named Phoneutria nigriventer tachykinin peptides PnTkPs (Pimenta et al., 2005). The PnTkPs display a variation of post-translational modifications such as proteolysis, C-terminal amidation, and cyclization (Pimenta et al., 2005). The new procedure also resulted in the discovery of two new structural families of Phoneutria peptides (Penaforte et al., 2000; Richardson et al., 2006). The family of $4 \mathrm{kDa}$
1 10

$\begin{array}{ll}\text { PnTkP-I } & \text { QKKDKKD } \\ \text { PnTkP-II } & \text { QKKDKKDK } \\ \text { PnTkP-III } & \text { QKKDKKDR } \\ \text { PnTkP-IV } & \text { QKKDKKDKF } \\ \text { PnTkP-V } & \text { QKKDKKDRF } \\ \text { PnTkP-VI } & \text { QKKDRFLGLM } \\ \text { PnTkP-VII } & \text { QKKDRFLGLF } \\ \text { PnTkP-VIII } & \text { QKKDKKDRFY } \\ \text { PnTkP-IX } & \text { QKKDKDRFYGLM } \\ \text { PnTkP-X } & \text { QKKDKKDKFYGLM } \\ \text { PnTkP-XI } & \text { QKNDKKDRFYGLM } \\ \text { PnTkP-XII } & \text { QKKDKKDKFYGLF } \\ \text { PnTkP-XIII } & \text { QKKDKKDRFYGLM } \\ \text { PnTkP-XIV } & \text { QKKDKKDRFYGLF } \\ \text { PnTkP-XV } & \text { QKKDKKDRFPNGLV }\end{array}$

Fig. 10. Amino acid sequences of the tachykinin family identified in the Phoneutria nigriventer venom.

toxins targets Cav channels (Lucio et al., 2008). Two different isoforms PnTx27C4 and PnTx26AN0C3 were isolated and characterized. Both peptides share $92 \%$ sequence identity. These toxins produce spastic paralysis and death in mice when injected intracerebral. Three very similar peptides, PnTx13C3, PnTx24An0C3 and PnTx24An0C4, were identified in this fraction. These peptides share $79 \%$ amino acid sequence identity. Similar to the PhTx 4 toxins, these $3.5 \mathrm{kDa}$ toxins are very toxic to houseflies and induce no observable toxic effects in mice by i.c. injection (Richardson et al., 2006).

Two proteases could be identified in the Phoneutria nigiriventer venom. They were named proteinase PN44 and proteinase PN47. The complete amino acid sequence of the PN47 and the N-terminal sequence of have been determined. Both proteases are serine proteases 


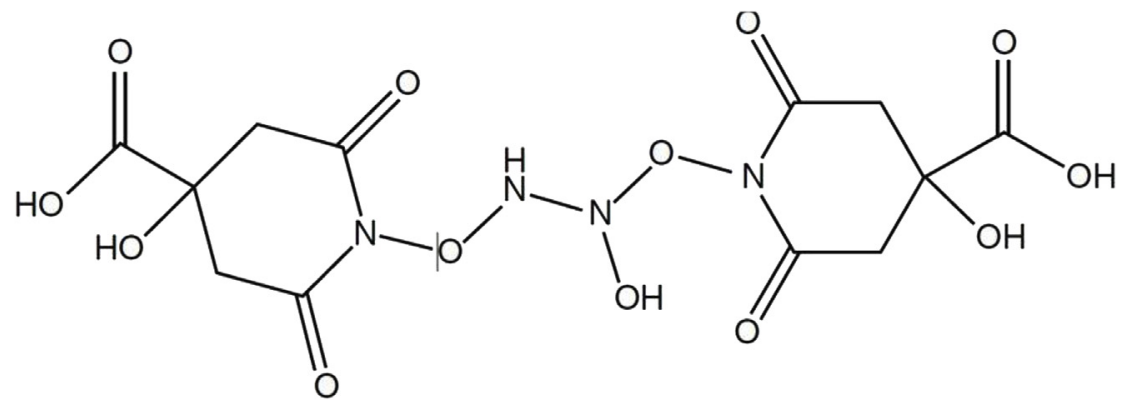

Fig. 11. Proposed structure of nigriventrine (Gomes et al., 2011).

belonging to the peptidase S1 family. It has been suggested that the endogenous proteolytic enzymes in the venom may be responsible for the post-translational modification observed in some of the venom components (de Lima et al., 2016).

\section{Non-protein $P$. nigirventer venom components}

In addition, a novel non-protein low-molecular-mass neurotoxin named nigriventrine was isolated from the hydrophilic fractions obtained from the venom by RP-HPLC purifications (Gomes et al., 2011). Nigriventrine, a piperidine derivative, has neuroactive properties and causes convulsion when injected in mice, intracerebrally or peripherally by intravenous injection (i.v.). Its structure was determined and it was characterized as a hydroxyl-hydrazyldioxopiperidine (Fig. 11) (Gomes et al., 2011).

\section{Conclusion and future directions}

It is estimated that venom of $P$. nigriventer is complex cocktail of more than 150 peptides/protein components (Richardson et al., 2006). Considering that only about 41 toxins were pharmacologically and/or chemically characterized so far, a wide diversity of new molecules, with possible different biological targets and activities, remains to be discovered in this venom. The studies conducted with $P$. nigriventer venom revealed several toxins acting on sodium, calcium, and TRPA channels, while other showed interesting activity on NMDA, cannabinoid or opioid receptors. Some of them have shown biotechnological and therapeutic potential, for example, by enhancing erectile function, acting as analgesics or insecticides. Although much of the richness and diversity of active peptides of Phoneutria nigriventer venom has been revealed many more remains to be discovered. For instance, a comprehensive transcriptomic study of this spider would greatly complement the proteomic work done so far. Furthermore, since at present not a single structure of a Phoneutria venom peptide has been elucidated many structural questions remain unanswered. Determination of the structures is a requisite for careful designed mutagenesis studies. Therefore, further exploration of the characterized Phoneutria toxins necessitate advancing on the structural knowledge of these peptides. It is interesting that up to now, the venom of Phoneutria nigriventer has been intensively investigated for drug discovery but only few studies have focussed on the insecticidal properties of the venom components. It can be anticipated that also within the venom of Phoneutria nigriventer peptides can be found with a potential as lead for the development of novel insecticidal agents. It is clear that the complex biochemical and pharmacological diversity of this venom has not yet been unravelled to the fullest and thus it can be expected in the years to come that many new peptides will be characterized as promising pharmacological tools, as lead compounds for drug development and as novel insecticidal agents.

\section{Acknowledgements}

M.E. de Lima received grants from Brazilian Agencies FAPEMIG (Fundação de Amparo à Pesquisa do Estado de Minas Gerais), CAPES (Coordenação de Aperfeiçoamento de Pessoal de Nível Superior) and CNPq (Conselho Nacional de Desenvolvimento Científico e Tecnológico).

\section{Transparency document}

Transparency document related to this article can be found online at https://doi.org/10.1016/j.toxicon.2018.07.008.

\section{References}

Agostini, R.M., do Nascimento Pinheiro, A.C., Binda, N.S., Romano Silva, M.A., do Nascimento Cordeiro, M., Richardson, M., Sena Guimaraes, A.L., Gomez, M.V., 2011 Phoneutria spider toxins block ischemia-induced glutamate release and neuronal death of cell layers of the retina. Retina 31 (7), 1392-1399 (Philadelphia, Pa ).

Almeida, A.P., Andrade, A.B., Ferreira, A.J., Pires, A.C.G., Damasceno, D.D., Alves, M.N.M., Gomes, E.R.M., Kushmerick, C., Lima, R.F., Prado, M.A.M., Prado, V.F., Richardson, M., Cordeiro, M.N., Guatimosim, S., Gomez, M., 2011.

Antiarrhythmogenic effects of a neurotoxin from the spider Phoneutria nigriventer. Toxicon 57 (2), 217-224.

Araujo, D.A., Cordeiro, M.N., Diniz, C.R., Beirao, P.S., 1993. Effects of a toxic fraction, PhTx2, from the spider Phoneutria nigriventer on the sodium current. N. Schmied. Arch. Pharmacol. 347 (2), 205-208.

Becker, S., Prusak-Sochaczewski, E., Zamponi, G., Beck-Sickinger, A.G., Gordon, R.D., French, R.J., 1992. Action of derivatives of $\mu$-conotoxin GIIIA on sodium channels. Single amino acid substitutions in the toxin separately affect association and dissociation rates. Biochemistry 31 (35), 8229-8238.

Binda, N.S., Carayon, C.P.P., Agostini, R.M., Pinheiro, ACdN., Cordeiro, M.N., Silva, M.A.R., Silva, J.F., Pereira, E.M.R., da Silva Junior, C.A., de Castro Junior, C.J., Guimaraes, A.L.S., Gomez, M.V., 2016. PhTx3-4, a spider toxin calcium channel blocker, reduces NMDA-induced injury of the retina. Toxins 8 (3).

Bucaretchi, F., Mello, S.M., Vieira, R.J., Mamoni, R.L., Blotta, M.H.S.L., Antunes, E. Hyslop, S., 2008. Systemic envenomation caused by the wandering spider Phoneutria nigriventer, with quantification of circulating venom. Clin. Toxicol. 46 (9), 885-889.

Bucherl, W., 1953. Comparison of the action of gland extracts and pure venom of Phoneutria nigriventer Keyserling 1891. Mem. Inst. Butantan (Sao Paulo) 25 (2), 1-21.

Bucherl, W., 1969. Biology and venoms of the most important South american spiders of the genera Phoneutria, loxosceles, Lycosa, and latrodectus. Am. Zool. 9 (1), 157-159.

Cardoso, F.C., Lewis, R.J., 2018 Jun. Sodium channels and pain: from toxins to therapies. Br. J. Pharmacol. 175 (12), 2138-2157.

Cardoso, F.C., Pacifico, L.G., Carvalho, D.C., Victoria, J.M.N., Neves, A.L.G., ChavezOlortegui, C., Gomez, M.V., Kalapothakis, E., 2003. Molecular cloning and characterization of Phoneutria nigriventer toxins active on calcium channels. Toxicon 41 (7), 755-763.

Carneiro, A.M.D., Kushmerick, C., Koenen, J., Arndt, M.H.L., Cordeiro, M.N., ChavezOlortegui, C., Diniz, C.R., Gomez, M.V., Kalapothakis, E., Prado, M.A.M., Prado, V.F., 2003. Expression of a functional recombinant Phoneutria nigriventer toxin active on $\mathrm{K}$ + channels. Toxicon 41 (3), 305-313.

Cassola, A.C., Jaffe, H., Fales, H.M., Afeche, S.C., Magnoli, F., Cipolla-Neto, J., 1998. omega-Phonetoxin-IIA: a calcium channel blocker from the spider Phoneutria nigriventer. Pflueg. Arch. Eur. J. Physiol. 436 (4), 545-552.

Castro-Junior, C.J., Milano, J., Souza, A.H., Silva, J.F., Rigo, F.K., Dalmolin, G., Cordeiro, M.N., Richardson, M., Barros, A.G.A., Gomez, R.S., Silva, M.A.R., Kushmerick, C., Ferreira, J., Gomez, M.V., 2013. Phalpha1beta toxin prevents capsaicin-induced nociceptive behavior and mechanical hypersensitivity without acting on TRPV1 channels. Neuropharmacology 71, 237-246.

Catterall, W.A., Cestele, S., Yarov-Yarovoy, V., Yu, F.H., Konoki, K., Scheuer, T., 2007 Voltage-gated ion channels and gating modifier toxins. Toxicon 49 (2), 124-141.

Chassagnon, I.R., McCarthy, C.A., Chin, Y.K., Pineda, S.S., Keramidas, A., Mobli, M., Pham, V., De Silva, T.M., Lynch, J.W., Widdop, R.E., Rash, L.D., King, G.F., 2017. Potent neuroprotection after stroke afforded by a double-knot spider-venom peptide that inhibits acid-sensing ion channel 1a. Proc. Natl. Acad. Sci. U. S. A. 114 (14), 
$3750-3755$

Cordeiro, MdN., de Figueiredo, S.G., Valentim, AdC., Diniz, C.R., von Eickstedt, V.R., Gilroy, J., Richardson, M., 1993. Purification and amino acid sequences of six Tx3 type neurotoxins from the venom of the Brazilian 'armed' spider Phoneutria nigriventer (Keys). Toxicon 31 (1), 35-42.

Cordeiro, MdN., Diniz, C.R., Valentim, AdC., von Eickstedt, V.R., Gilroy, J., Richardson, M., 1992. The purification and amino acid sequences of four Tx2 neurotoxins from the venom of the Brazilian 'armed' spider Phoneutria nigriventer (Keys). FEBS Lett. 310 (2), 153-156.

Cruz-Hofling, M.A., Tavares, J.C., Rapôso, C., 2016. Phoneutria nigriventer venom: action in the central nervous system. In: Gopalkrishnakone, P. (Ed.), Spider Venoms, Toxinology. Springer, pp. 176-197.

da Fonseca Pacheco, D., Freitas, A.C.N., Pimenta, A.M.C., Duarte, I.D.G., de Lima, M.E., 2016. A spider derived peptide, PnPP-19, induces central antinociception mediated by opioid and cannabinoid systems. J. Venom. Anim. Toxins Incl. Trop. Dis. 22, 34.

Dalmolin, G.D., Bannister, K., Goncalves, L., Sikandar, S., Patel, R., Cordeiro, MdN., Gomez, M.V., Ferreira, J., Dickenson, A.H., 2017. Effect of the spider toxin Tx3-3 on spinal processing of sensory information in naive and neuropathic rats: an in vivo electrophysiological study. Pain Rep. 2 (4), e610.

de Figueiredo, S.G., de Lima, M.E., Nascimento Cordeiro, M., Diniz, C.R., Patten, D., Halliwell, R.F., Gilroy, J., Richardson, M., 2001. Purification and amino acid sequence of a highly insecticidal toxin from the venom of the brazilian spider Phoneutria nigriventer which inhibits NMDA-evoked currents in rat hippocampal neurones. Toxicon 39 (2-3), 309-317.

De Lima, M.E., Figueiredo, S.G., Pimenta, A.M.C., Santos, D.M., Borges, M.H., Cordeiro, M.N., Richardson, M., Oliveira, L.C., Stankiewicz, M., Pelhate, M., 2007. Peptides of arachnid venoms with insecticidal activity targeting sodium channels. Comparat. Biochem. Physiol. Toxicol. Pharmacol. 146 (1-2), 264-279.

de Lima, M.E., Figueiredo, S.G., Matavel, A., Nunes, K.P., da Silva, C.N., Almeida, F.M., Diniz, M.R.V., Cordeiro, M.N., Stankiewicz, M., Beirao, P.S., 2016. Phoneutria nigriventer venom and toxins: a review. In: Gopalkrishnakone, P. (Ed.), Spider Venoms, Toxinology. Springer, pp. 71-99.

de Lima, M.E., Stankiewicz, M., Hamon, A., de Figueiredo, S.G., Cordeiro, M.N., Diniz, C.R., Martin-Eauclaire, M.F., Pelhate, M., 2002. The toxin Tx4(6-1) from the spider Phoneutria nigriventer slows down $\mathrm{Na}+$ current inactivation in insect CNS via binding to receptor site 3. J. Insect Physiol. 48 (1), 53-61.

Deuis, J.R., Mueller, A., Israel, M.R., Vetter, I., 2017. The pharmacology of voltage-gated sodium channel activators. Neuropharmacology $127,87-108$.

Diniz, C., 1963. Separation of proteins and characterization of active substances in the venom of the Brazilian spiders. An. Acad. Bras. Cienc. 35, 283-291.

Diniz, C.R., Cordeiro, MdN., Junor, L.R., Kelly, P., Fischer, S., Reimann, F., Oliveira, E.B., Richardson, M., 1990. The purification and amino acid sequence of the lethal neurotoxin Tx1 from the venom of the Brazilian 'armed' spider Phoneutria nigriventer. FEBS Lett. 263 (2), 251-253.

Diniz, M.R., Paine, M.J., Diniz, C.R., Theakston, R.D., Crampton, J.M., 1993. Sequence of the cDNA coding for the lethal neurotoxin Tx1 from the Brazilian "armed" spider Phoneutria nigriventer predicts the synthesis and processing of a preprotoxin. J. Biol. Chem. 268 (21), 15340-15342.

Diniz, M.R.V., Theakston, R.D.G., Crampton, J.M., Nascimento Cordeiro, Md, Pimenta, A.M.C., De Lima, M.E., Diniz, C.R., 2006. Functional expression and purification of recombinant Tx1, a sodium channel blocker neurotoxin from the venom of the Brazilian "armed" spider, Phoneutria nigriventer. Protein Expr. Purif. 50 (1), 18-24.

Dos Santos, R.G., Van Renterghem, C., Martin-Moutot, N., Mansuelle, P., Cordeiro, M.N., Diniz, C.R., Mori, Y., De Lima, M.E., Seagar, M., 2002. Phoneutria nigriventer omegaphonetoxin IIA blocks the Cav2 family of calcium channels and interacts with omegaconotoxin-binding sites. J. Biol. Chem. 277 (16), 13856-13862.

Emerich, B.L., Ferreira, R.C.M., Cordeiro, M.N., Borges, M.H., Pimenta, A.M.C., Figueiredo, S.G., Duarte, I.D.G., de Lima, M.E., 2016. $\delta$-Ctenitoxin-Pn1a, a peptide from Phoneutria nigriventer spider venom, shows antinociceptive effect involving opioid and cannabinoid systems, in rats. Toxins 8 (4).

Escoubas, P., 2006. Molecular diversification in spider venoms: a web of combinatorial peptide libraries. Mol. Divers. 10 (4), 545-554.

Figueiredo, S.G., Garcia, M.E., Valentim, A.C., Cordeiro, M.N., Diniz, C.R., Richardson, M., 1995. Purification and amino acid sequence of the insecticidal neurotoxin Tx4(61) from the venom of the 'armed' spider Phoneutria nigriventer (Keys). Toxicon 33 (1), 83-93.

Fitches, E.C., Pyati, P., King, G.F., Gatehouse, J.A., 2012. Fusion to snowdrop lectin magnifies the oral activity of insecticidal omega-Hexatoxin-Hvla peptide by enabling its delivery to the central nervous system. PLoS One 7 (6), e39389.

Freitas, A.C.N., Pacheco, D.F., Machado, M.F.M., Carmona, A.K., Duarte, I.D.G., de Lima, M.E., 2016. PnPP-19, a spider toxin peptide, induces peripheral antinociception through opioid and cannabinoid receptors and inhibition of neutral endopeptidase. Br. J. Pharmacol. 173 (9), 1491-1501.

Freitas, A.C.N., Peigneur, S., Macedo, F.H.P., Menezes-Filho, J.E., Millns, P., Medeiros, L.F., Arruda, M.A., Cruz, J., Holliday, N.D., Tytgat, J., Hathway, G., de Lima, M.E., 2018. The peptide PnPP-19, a spider toxin derivative, activates $\mu$-opioid receptors and modulates calcium channels. Toxins 10 (1).

Freitas, A.C., Silva, G.C., Pacheco, D.F., Pimenta, A.M., Lemos, V.S., Duarte, I.D., de Lima, M.E., 2017 Apr 1. The synthetic peptide PnPP-19 induces peripheral antinociception via activation of NO/cGMP/KATP pathway: role of eNOS and nNOS. Nitric Oxide 64, 31-38.

French, R.J., Yoshikami, D., Sheets, M.F., Olivera, B.M., 2010. The tetrodotoxin receptor of voltage-gated sodium channels-perspectives from interactions with micro-conotoxins. Mar. Drugs 8 (7), 2153-2161.

Gomes, P.C., de Souza, B.M., Dias, N.B., Cesar-Tognoli, L.M.M., Silva, L.C., Tormena, C.F., Rittner, R., Richardson, M., Cordeiro, M.N., Palma, M.S., 2011. Nigriventrine: a low molecular mass neuroactive compound from the venom of the spider Phoneutria nigriventer. Toxicon 57 (2), 266-274.

Gomez, M.V., Kalapothakis, E., Guatimosim, C., Prado, M.A.M., 2002. Phoneutria nigriventer venom: a cocktail of toxins that affect ion channels. Cell. Mol. Neurobiol. 22
(5-6), 579-588.

Gomez, R.S., Casali, T.A., Romano-Silva, M.A., Cordeiro, M.N., Diniz, C.R., MoraesSantos, T., Prado, M.A., Gomez, M.V., 1995. The effect of PhTx3 on the release of 3Hacetylcholine induced by tityustoxin and potassium in brain cortical slices and myenteric plexus. Neurosci. Lett. 196 (1-2), 131-133.

Goncaves, J.M., Ferreira, J., Prado, M.A.M., Cordeiro, M.N., Richardson, M., Pinheiro, ACdN., Silva, M.A.R., Junior, CJdC., Souza, A.H., Gomez, M.V., 2011. The effect of spider toxin PhTx3-4, omega-conotoxins MVIIA and MVIIC on glutamate uptake and on capsaicin-induced glutamate release and $[\mathrm{Ca} 2+] \mathrm{i}$ in spinal cord synaptosomes. Cell. Mol. Neurobiol. 31 (2), 277-283.

Green, B.R., Bulaj, G., Norton, R.S., 2014. Structure and function of mu-conotoxins, peptide-based sodium channel blockers with analgesic activity. Future Med. Chem. 6 (15), 1677-1698.

Green, B.R., Olivera, B.M., 2016. Venom peptides from cone snails: pharmacological probes for voltage-gated sodium channels. Curr. Top. Membr. 78, 65-86.

Guatimosim, C., Romano-Silva, M.A., Cruz, J.S., Beirao, P.S., Kalapothakis, E., MoraesSantos, T., Cordeiro, M.N., Diniz, C.R., Gomez, M.V., Prado, M.A., 1997. A toxin from the spider Phoneutria nigriventer that blocks calcium channels coupled to exocytosis. Br. J. Pharmacol. 122 (3), 591-597.

Hauke, T.J., Herzig, V., 2017. Dangerous arachnids-Fake news or reality? Toxicon 138, 173-183.

Herzig, V., 2016. Create guidelines for characterization of venom peptides. Toxins 8 (9).

Herzig, V., John Ward, R., Ferreira dos Santos, W., 2002. Intersexual variations in the venom of the Brazilian 'armed' spider Phoneutria nigriventer (Keyserling, 1891). Toxicon 40 (10), 1399-1406.

Herzig, V., King, G.F., 2015. The cystine knot is responsible for the exceptional stability of the insecticidal spider toxin omega-hexatoxin-hvla. Toxins 7 (10), 4366-4380.

Herzig, V., Wood, D.L., Newell, F., Chaumeil, P.A., Kaas, Q., Binford, G.J., Nicholson, G.M., Gorse, D., King, G.F., 2011. ArachnoServer 2.0, an updated online resource for spider toxin sequences and structures. Nucleic Acids Res. 39 (Database issue), D653-D657.

Israel, M.R., Tay, B., Deuis, J.R., Vetter, I., 2017. Sodium channels and venom peptide pharmacology. Adv. Pharmacol. 79, 67-116.

Kalapothakis, E., Penaforte, C.L., Beirao, P.S., Romano-Silva, M.A., Cruz, J.S., Prado, M.A., Guimaraes, P.E., Gomez, M.V., Prado, V.F., 1998a. Cloning of cDNAS encoding neurotoxic peptides from the spider Phoneutria nigriventer. Toxicon 36 (12), 1843-1850.

Kalapothakis, E., Penaforte, C.L., Leao, R.M., Cruz, J.S., Prado, V.F., Cordeiro, M.N., Diniz, C.R., Romano-Silva, M.A., Prado, M.A., Gomez, M.V., Beirao, P.S., 1998b. Cloning, cDNA sequence analysis and patch clamp studies of a toxin from the venom of the armed spider (Phoneutria nigriventer). Toxicon 36 (12), 1971-1980.

Khoo, K.K., Wilson, M.J., Smith, B.J., Zhang, M.-M., Gulyas, J., Yoshikami, D., Rivier, J.E., Bulaj, G., Norton, R.S., 2011. Lactam-stabilized helical analogues of the analgesic $\mu-$ conotoxin KIIIA. J. Med. Chem. 54 (21), 7558-7566.

King, G.F., Hardy, M.C., 2013. Spider-venom peptides: structure, pharmacology, and potential for control of insect pests. Annu. Rev. Entomol. 58, 475-496.

Klint, J.K., Senff, S., Rupasinghe, D.B., Er, S.Y., Herzig, V., Nicholson, G.M., King, G.F., 2012. Spider-venom peptides that target voltage-gated sodium channels: pharmacological tools and potential therapeutic leads. Toxicon 60 (4), 478-491.

Kushmerick, C., Kalapothakis, E., Beirao, P.S., Penaforte, C.L., Prado, V.F., Cruz, J.S., Diniz, C.R., Cordeiro, M.N., Gomez, M.V., Romano-Silva, M.A., Prado, M.A., 1999 Phoneutria nigriventer toxin Tx3-1 blocks A-type $\mathrm{K}+$ currents controlling Ca2 + oscillation frequency in GH3 cells. J. Neurochem. 72 (4), 1472-1481.

King, G.F., Gentz, M.C., Escoubas, P., Nicholson, G.M., 2008 Aug 1. Arational nomenclature for naming peptide toxins from spiders and other venomous animals. Toxicon 52 (2), 264-276.

Leao, R.M., Cruz, J.S., Diniz, C.R., Cordeiro, M.N., Beirao, P.S., 2000. Inhibition of neuronal high-voltage activated calcium channels by the omega-Phoneutria nigriventer Tx3-3 peptide toxin. Neuropharmacology 39 (10), 1756-1767.

Leipold, E., Hansel, A., Borges, A., Heinemann, S.H., 2006. Subtype specificity of scorpion $\beta$-toxin Tz1 interaction with voltage-gated sodium channels is determined by the pore loop of domain 3. Mol. Pharmacol. 70 (1), 340-347.

Lucio, A.D., Campos, F.V., Richardson, M., Cordeiro, M.N., Mazzoni, M.S.C., de Lima, M.E., Pimenta, A.M.C., Bemquerer, M.P., Figueiredo, S.G., Gomes, P.C., Beirao, P.S.L. 2008. A new family of small ( $4 \mathrm{kDa}$ ) Neurotoxins from the venoms of spiders of the genus Phoneutria. Protein Pept. Lett. 15 (7), 700-708.

Mafra, R.A., Figueiredo, S.G., Diniz, C.R., Cordeiro, M.N., Cruz, J.D., De Lima, M.E., 1999. PhTx4, a new class of toxins from Phoneutria nigriventer spider venom, inhibits the glutamate uptake in rat brain synaptosomes. Brain Res. 831 (1-2), 297-300.

Martin-Moutot, N., Mansuelle, P., Alcaraz, G., Dos Santos, R.G., Cordeiro, M.N., De Lima, M.E., Seagar, M., Van Renterghem, C., 2006. Phoneutria nigriventer toxin 1: a novel, state-dependent inhibitor of neuronal sodium channels that interacts with micro conotoxin binding sites. Mol. Pharmacol. 69 (6), 1931-1937.

Matavel, A., Cruz, J.S., Penaforte, C.L., Araujo, D.A.M., Kalapothakis, E., Prado, V.F., Diniz, C.R., Cordeiro, M.N., Beirao, P.S.L., 2002. Electrophysiological characterization and molecular identification of the Phoneutria nigriventer peptide toxin PnTx2-6. FEBS Lett. 523 (1-3), 219-223.

Matavel, A., Fleury, C., Oliveira, L.C., Molina, F., de Lima, M.E., Cruz, J.S., Cordeiro, M.N., Richardson, M., Ramos, C.H., Beirao, P.S., 2009. Structure and activity analysis of two spider toxins that alter sodium channel inactivation kinetics. Biochemistry 48 (14), 3078-3088.

McGivern, J.G., 2007. Ziconotide: a review of its pharmacology and use in the treatment of pain. Neuropsychiatric Dis. Treat. 3 (1), 69-85.

Miranda, D.M., Romano-Silva, M.A., Kalapothakis, E., Diniz, C.R., Cordeiro, M.N., Moraes-Santos, T., De Marco, L., Prado, M.A., Gomez, M.V., 2001. Spider neurotoxins block the beta scorpion toxin-induced calcium uptake in rat brain cortical synaptosomes. Brain Res. Bull. 54 (5), 533-536.

Nicholson, G.M., 2007. Insect-selective spider toxins targeting voltage-gated sodium channels. Toxicon 49 (4), 490-512.

Nicholson, G.M., Little, M.J., Birinyi-Strachan, L.C., 2004. Structure and function of $\delta$ - 
atracotoxins: lethal neurotoxins targeting the voltage-gated sodium channel. Toxicon 43 (5), 587-599.

Nicoletti, N.F., Erig, T.C., Zanin, R.F., Roxo, M.R., Ferreira, N.P., Gomez, M.V., Morrone, F.B., Campos, M.M., 2017. Pre-clinical evaluation of voltage-gated calcium channel blockers derived from the spider P.nigriventer in glioma progression. Toxicon 129, 58-67.

Nunes, K.P., Cordeiro, M.N., Richardson, M., Borges, M.N., Diniz, S.O.F., Cardoso, V.N. Tostes, R., De Lima, M.E., Webb, R.C., Leite, R., 2010. Nitric oxide-induced vasorelaxation in response to PnTx2-6 toxin from Phoneutria nigriventer spider in rat cavernosal tissue. J. Sex. Med. 7 (12), 3879-3888.

Nunes, K.P., Costa-Goncalves, A., Lanza, L.F., Cortes, S.F., Cordeiro, M.N., Richardson, M., Pimenta, A.M.C., Webb, R.C., Leite, R., De Lima, M.E., 2008. Tx2-6 toxin of the Phoneutria nigriventer spider potentiates rat erectile function. Toxicon 51 (7), $1197-1206$.

Nunes, K.P., Toque, H.A., Borges, M.H., Richardson, M., Webb, R.C., de Lima, M.E., 2012a. Erectile function is improved in aged rats by PnTx2-6, a toxin from Phoneutria nigriventer spider venom. J. Sex. Med. 9 (10), 2574-2581.

Nunes, K.P., Wynne, B.M., Cordeiro, M.N., Borges, M.H., Richardson, M., Leite, R., DeLima, M.E., Webb, R.C., 2012b. Increased cavernosal relaxation by Phoneutria nigriventer toxin, PnTx2-6, via activation at NO/cGMP signaling. Int. J. Impot. Res. 24 (2), 69-76.

Oliveira, L.C., De Lima, M.E., Pimenta, A.M.C., Mansuelle, P., Rochat, H., Cordeiro, M.N., Richardson, M., Figueiredo, S.G., 2003. PnTx4-3, a new insect toxin from Phoneutria nigriventer venom elicits the glutamate uptake inhibition exhibited by PhTx4 toxic fraction. Toxicon 42 (7), 793-800.

Oliveira, S.M., Silva, C.R., Trevisan, G., Villarinho, J.G., Cordeiro, M.N., Richardson, M., Borges, M.H., Castro Jr., C.J., Gomez, M.V., Ferreira, J., 2016. Antinociceptive effect of a novel armed spider peptide Tx3-5 in pathological pain models in mice. Pflueg. Arch. Eur. J. Physiol. 468 (5), 881-894.

Paiva, A.L., Matavel, A., Peigneur, S., Cordeiro, M.N., Tytgat, J., Diniz, M.R., de Lima, M.E., 2016. Differential effects of the recombinant toxin PnTx4(5-5) from the spider Phoneutria nigriventer on mammalian and insect sodium channels. Biochimie 121 326-335.

Palhares, M.R., Silva, J.F., Rezende, M.J.S., Santos, D.C., Silva-Junior, C.A., Borges, M.H., Ferreira, J., Gomez, M.V., Castro-Junior, C.J., 2017. Synergistic antinociceptive effect of a calcium channel blocker and a TRPV1 blocker in an acute pain model in mice. Life Sci. 182, 122-128.

Peigneur, S., Cologna, C.T., Cremonez, C.M., Mille, B.G., Pucca, M.B., Cuypers, E., Arantes, E.C., Tytgat, J., 2015. A gamut of undiscovered electrophysiological effects produced by Tityus serrulatus toxin 1 on NaV-type isoforms. Neuropharmacology 95, 269-277.

Peigneur, S., Sevcik, C., Tytgat, J., Castillo, C., D'Suze, G., 2012. Subtype specificity interaction of bactridines with mammalian, insect and bacterial sodium channels under voltage clamp conditions. FEBS J. 279 (21), 4025-4038.

Penaforte, C.L., Prado, V.F., Prado, M.A., Romano-Silva, M.A., Guimaraes, P.E., De Marco, L., Gomez, M.V., Kalapothakis, E., 2000. Molecular cloning of cDNAs encoding insecticidal neurotoxic peptides from the spider Phoneutria nigriventer. Toxicon 38 (10), $1443-1449$.

Pimenta, A.M.C., Rates, B., Bloch, C., Gomes, P.C., Santoro, M.M., de Lima, M.E., Richardson, M., Cordeiro, M.D., 2005. Electrospray ionization quadrupole time-offlight and matrix-assisted laser desorption/ionization tandem time-of-flight mass spectrometric analyses to solve micro-heterogeneity in post-translationally modified peptides from Phoneutria nigriventer (Aranea, Ctenidae) venom. Rapid Commun. Mass Spectrom. 19 (1), 31-37.

Pineda, S.S., Chaumeil, P.A., Kunert, A., Kaas, Q., Thang, M.W.C., Li, L., Nuhn, M., Herzig, V., Saez, N.J., Cristofori-Armstrong, B., Anangi, R., Senff, S., Gorse, D., King, G.F. 2018 Mar 15. ArachnoServer 3.0: an online resource for automated discovery, analysis and annotation of spider toxins. Bioinformatics 34 (6), 1074-1076.

Pineda, S.S., Undheim, E.A., Rupasinghe, D.B., Ikonomopoulou, M.P., King, G.F., 2014. Spider venomics: implications for drug discovery. Future Med. Chem. 6 (15), 1699-1714.

Pinheiro, ACdN., da Silva, A.J., Prado, M.A.M., Cordeiro, MdN., Richardson, M., Batista, M.C., de Castro Junior, C.J., Massensini, A.R., Guatimosim, C., Romano-Silva, M.A., Kushmerick, C., Gomez, M.V., 2009. Phoneutria spider toxins block ischemia-induced glutamate release, neuronal death, and loss of neurotransmission in hippocampus. Hippocampus 19 (11), 1123-1129.

Pinheiro, A.C.N., Gomez, R.S., Massensini, A.R., Cordeiro, M.N., Richardson, M., RomanoSilva, M.A., Prado, M.A.M., De Marco, L., Gomez, M.V., 2006. Neuroprotective effect on brain injury by neurotoxins from the spider Phoneutria nigriventer. Neurochem. Int. 49 (5), 543-547.

Prado, M.A., Guatimosim, C., Gomez, M.V., Diniz, C.R., Cordeiro, M.N., Romano-Silva, M.A., 1996. A novel tool for the investigation of glutamate release from rat cerebrocortical synaptosomes: the toxin Tx3-3 from the venom of the spider Phoneutria nigriventer. Biochem. J. 314 (Pt 1), 145-150.

Prashanth, J.R., Brust, A., Jin, A.-H., Alewood, P.F., Dutertre, S., Lewis, R.J., 2014. Cone snail venomics: from novel biology to novel therapeutics. Future Med. Chem. 6 (15), 1659-1675.

Raposo, C., Bjorklund, U., Kalapothakis, E., Biber, B., Alice da Cruz-Hofling, M., Hansson, E., 2016. Neuropharmacological effects of Phoneutria nigriventer venom on astrocytes. Neurochem. Int. 96, 13-23.

Ravelli, K.G., Ramos, AdT., Goncalves, L.B., Magnoli, F.C., Troncone, L.R.P., 2017. Phoneutria nigriventer spider toxin Tx2-6 induces priapism in mice even after cavernosal denervation. Toxicon 130, 29-34.

Reis, H.J., Prado, M.A., Kalapothakis, E., Cordeiro, M.N., Diniz, C.R., De Marco, L.A.,
Gomez, M.V., Romano-Silva, M.A., 1999. Inhibition of glutamate uptake by a polypeptide toxin (Phoneutriatoxin 3-4) from the spider Phoneutria nigriventer. Biochem. J. 343 (Pt 2), 413-418.

Rezende Jr., L., Cordeiro, M.N., Oliveira, E.B., Diniz, C.R., 1991. Isolation of neurotoxic peptides from the venom of the 'armed' spider Phoneutria nigriventer. Toxicon 29 (10), 1225-1233.

Richardson, M., Pimenta, A.M.C., Bemquerer, M.P., Santoro, M.M., Beirao, P.S.L., Lima, M.E., Figueiredo, S.G., Bloch Jr., C., Vasconcelos, E.A.R., Campos, F.A.P., Gomes, P.C., Cordeiro, M.N., 2006. Comparison of the partial proteomes of the venoms of Brazilian spiders of the genus Phoneutria. Comparat. Biochem. Physiol. Toxicol. Pharmacol.: CBP 142 (3-4), 173-187.

Rigo, F.K., Rossato, M.F., Trevisan, G., De Pra, S.D.-T., Ineu, R.P., Duarte, M.B., de Castro Junior, C.J., Ferreira, J., Gomez, M.V., 2017. PhKv a toxin isolated from the spider venom induces antinociception by inhibition of cholinesterase activating cholinergic system. Scandinavian J. Pain 17, 203-210.

Santos, R.G., Diniz, C.R., Cordeiro, M.N., De Lima, M.E., 1999. Binding sites and actions of Tx1, a neurotoxin from the venom of the spider Phoneutria nigriventer, in Guinea pig ileum. Braz. J. Med. Biol. Res. 32 (12), 1565-1569 Revista brasileira de pesquisas medicas e biologicas.

Schenberg, S., Lima, F.A., 1966. Pharmacology of the polypeptides from the venom of the spider Phoneutria fera. Mem. Inst. Butantan (Sao Paulo) 33 (2), 627-638.

Silva, A.O., Peigneur, S., Diniz, M.R.V., Tytgat, J., Beirao, P.S.L., 2012. Inhibitory effect of the recombinant Phoneutria nigriventer Tx1 toxin on voltage-gated sodium channels. Biochimie 94 (12), 2756-2763.

Silva, C.N., Nunes, K.P., Torres, F.S., Cassoli, J.S., Santos, D.M., Almeida, F.D.M., Matavel, A., Cruz, J.S., Santos-Miranda, A., Nunes, A.D.C., Castro, C.H., Machado de Avila, R.A., Chavez-Olortegui, C., Lauar, S.S., Felicori, L., Resende, J.M., Camargos, ERdS., Borges, M.H., Cordeiro, M.N., Peigneur, S., Tytgat, J., de Lima, M.E., 2015a. PnPP-19, a synthetic and nontoxic peptide designed from a Phoneutria nigriventer toxin, potentiates erectile function via no/cGMP. J. Urol. 194 (5), 1481-1490.

Silva, F.R., Batista, E.M.L., Gomez, M.V., Kushmerick, C., Da Silva, J.F., Cordeiro, M.N., Vieira, L.B., Ribeiro, F.M., 2016. The Phoneutria nigriventer spider toxin, PnTx4-5-5, promotes neuronal survival by blocking NMDA receptors. Toxicon 112, 16-21.

Silva, R.B.M., Sperotto, N.D.M., Andrade, E.L., Pereira, T.C.B., Leite, C.E., de Souza, A.H. Bogo, M.R., Morrone, F.B., Gomez, M.V., Campos, M.M., 2015b. Spinal blockage of P/ Q- or N-type voltage-gated calcium channels modulates functional and symptomatic changes related to haemorrhagic cystitis in mice. Br. J. Pharmacol. 172 (3), 924-939.

Souza, A.H., Ferreira, J., Cordeiro, MdN., Vieira, L.B., De Castro, C.J., Trevisan, G., Reis, H., Souza, I.A., Richardson, M., Prado, M.A.M., Prado, V.F., Gomez, M.V., 2008. Analgesic effect in rodents of native and recombinant $\mathrm{Ph} \alpha 1 \beta$ toxin, a high-voltageactivated calcium channel blocker isolated from armed spider venom. Pain 140 (1), $115-126$.

Swartz, K.J., 2007 Feb. Tarantulatoxins interacting with voltage sensors in potassium channels. Toxicon 49 (2), 213-230.

Tonello, R., Fusi, C., Materazzi, S., Marone, I.M., De Logu, F., Benemei, S., Goncalves, M.C., Coppi, E., Castro-Junior, C.J., Gomez, M.V., Geppetti, P., Ferreira, J., Nassini, R., 2017. The peptide Phalphalbeta, from spider venom, acts as a TRPA1 channel antagonist with antinociceptive effects in mice. Br. J. Pharmacol. 174 (1), 57-69.

Tonello, R., Rigo, F., Gewehr, C., Trevisan, G., Pereira, E.M.R., Gomez, M.V., Ferreira, J., 2014. Action of Phalpha1beta, a peptide from the venom of the spider Phoneutria nigriventer, on the analgesic and adverse effects caused by morphine in mice. J. Pain: Official J. Am. Pain Soc. 15 (6), 619-631.

Torres, F.S., Silva, C.N., Lanza, L.F., Santos, A.V., Pimenta, A.M.C., De Lima, M.E., Diniz, M.R.V., 2010. Functional expression of a recombinant toxin - rPnTx2-6 - active in erectile function in rat. Toxicon 56 (7), 1172-1180.

Troncone, L.R.P., Georgiou, J., Hua, S.-Y., Elrick, D., Lebrun, I., Magnoli, F., Charlton, M.P., 2003. Promiscuous and reversible blocker of presynaptic calcium channels in frog and crayfish neuromuscular junctions from Phoneutria nigriventer spider venom. J. Neurophysiol. 90 (5), 3529-3537.

Van Der Haegen, A., Peigneur, S., Tytgat, J., 2011. Importance of position 8 in $\mu$-conotoxin KIIIA for voltage-gated sodium channel selectivity. FEBS J. 278 (18), 3408-3418.

Verma, D., Gupta, Y.K., Parashar, A., Ray, S.B., 2009. Differential expression of L- and Ntype voltage-sensitive calcium channels in the spinal cord of morphine + nimodipine treated rats. Brain Res. 1249, 128-134.

Vieira, L.B., Kushmerick, C., Hildebrand, M.E., Garcia, E., Stea, A., Cordeiro, M.N., Richardson, M., Gomez, M.V., Snutch, T.P., 2005. Inhibition of high voltage-activated calcium channels by spider toxin PnTx3-6. J. Pharmacol. Exp. Therapeut. 314 (3), 1370-1377.

Villanova, F.E., Andrade, E., Leal, E., Andrade, P.M., Borra, R.C., Troncone, L.R.P., Magalhaes, L., Leite, K.R.M., Paranhos, M., Claro, J., Srougi, M., 2009. Erection induced by Tx2-6 toxin of Phoneutria nigriventer spider: expression profile of genes in the nitric oxide pathway of penile tissue of mice. Toxicon 54 (6), 793-801.

Wanke, E., Zaharenko, A.J., Redaelli, E., Schiavon, E., 2009. Actions of sea anemone type 1 neurotoxins on voltage-gated sodium channel isoforms. Toxicon 54 (8), 1102-1111.

Yonamine, C.M., Troncone, L.R.P., Camillo, M.A.P., 2004. Blockade of neuronal nitric oxide synthase abolishes the toxic effects of Tx2-5, a lethal Phoneutria nigriventer spider toxin. Toxicon 44 (2), 169-172.

Zhang, M.-M., Han, T.S., Olivera, B.M., Bulaj, G., Yoshikami, D., 2010. -conotoxin KIIIA derivatives with divergent affinities versus efficacies in blocking voltage-gated sodium channels. Biochemistry 49 (23), 4804-4812.

Zhu, S., Peigneur, S., Gao, B., Lu, X., Cao, C., Tytgat, J., 2012. Evolutionary diversification of Mesobuthus a-scorpion toxins affecting sodium channels. Mol. Cell. Proteomics: MCP 11 (1), M111.012054. 